\title{
Comparison of the efficacy among multiple chemotherapeutic interventions combined with radiation therapy for patients with cervix cancer after surgery: A network meta-analysis
}

\author{
Lei Chang ${ }^{1}$ and Ruixia Guo ${ }^{1}$ \\ ${ }^{1}$ Department of Gynecology, The First Affiliated Hospital of Zhengzhou University, Zhengzhou, Henan 450000, China \\ Correspondence to: Lei Chang, email: nancy_lei7985@163.com \\ Keywords: cervix cancer, radiotherapy, chemotherapy, efficacy, network meta-analysis \\ Received: November 24, $2016 \quad$ Accepted: February 15, $2017 \quad$ Published: April 20, 2017 \\ Copyright: Chang et al. This is an open-access article distributed under the terms of the Creative Commons Attribution License \\ 3.0 (CC BY 3.0), which permits unrestricted use, distribution, and reproduction in any medium, provided the original author and \\ source are credited.
}

\section{ABSTRACT}

Background: Cervix cancer was the second most common cancer in female. However, there was no network meta-analysis (NMA) comparing the efficacy of the multiple chemotherapeutic interventions combined with radiation therapy in patients after operation.

Methods: Randomized controlled trials were retrieved from PubMed, Embase and Cochrane Library. Overall survival (OS), recurrence-free survival (RFS), incidence of recurrence and distant metastasis were the main outcomes, particularly 5-year OS and PFS were considered as primary outcomes. Furthermore, the hazard ratio (HR) or odds ratio (OR) and their $95 \%$ credible intervals (CrIs) were extracted. The surface under cumulative ranking curve (SUCRA) was also used in this NMA.

Results: A total of 39 eligible trials with 8,952 patients were included and 22 common chemotherapies were evaluated in this meta-analysis. For OS, cisplatin+fluo rouracil+hydroxyurea, fluorouracil+mitomycin $\mathrm{C}$, cisplatin and cisplatin+fluorouracil were better than placebo. As for RFS, cisplatin+fluorouracil, fluorouracil+mitomycin $C_{\text {, }}$ and cisplatin alone had the significant superiority compared with placebo. In terms of incidence of recurrence, the optimal drug combination was cisplatin+ifosfamide (0.93) based on SUCRA. Moreover, epirubicin (OR = 0.28, 95\% CrI: 0.08-0.91) was the only one had the distinguished potency in reducing the occurrence of distant metastasis with a SUCRA rank probability of 0.88 .

Conclusion: We recommended cisplatin+fluorouracil+hydroxyurea and cisplatin+docetaxel for their good efficacy in long term survival. Meanwhile, the combination of multiple drugs with different mechanisms worked better.

\section{INTRODUCTION}

Cervix cancer is caused by abnormal proliferation of cells with capacity of invasion, arising from the lower, narrow end of uterus to vagina. According to the definition of the National Cancer Institution of American National Institutes of Health, cervix cancer contains two main types, the squamous cell carcinoma originating from thin, flat cells that line the cervix and the adenocarcinoma arose from cells that make mucus and other fluids [1, $2]$. And it is the second most frequent cancer occurred in female worldwide, next to breast cancer, accounting for $7.9 \%$ cancer cases and $7.5 \%$ cancer deaths in female in 2012 [3, 4]. With the development of medical and healthcare system, the average 5-year survival rate of cervical cancer has reached $66 \%$ in developed countries, yet less than half patients from developing countries could live longer than 5 years $[5,6]$. The international biological study on etiology reported that human papillomavirus (HPV) was responsible for about $93 \%$ invasive cervical cancers [7]. Additionally, cigarette smoking, no matter active or passive, long-term use of oral contraceptives and multiple pregnancies are also high risk factors for cervix cancer $[8,9]$. 
On the fundamental of clinical examination instead of the surgical findings, the International Federation of Gynecology and Obstetrics (FIGO) divides the cervix cancer into five stages from 0 stage to IV stage [10]. Surgery is a common treatment, while the specific operation and scope for different FIGO stages are distinctive. Except for conization and hysterectomy, one of the traditional surgeries usually performed on stage IA microinvasive cancer, there are many methods of fertility preservation for cervical cancer, as radical vaginal trachelectomy [11]. Besides, radiotherapy is also an available way to treat cervical cancer and preserve reproductive function, splitting into external and internal radiation therapy, which are given depending on both type and stage of the cancer [12].

For further improvement of survival length and decreasing the incidence of recurrence and distant metastasis, chemotherapy is often combined with radiotherapy after surgery through multiple mechanisms exerting the synergistic effect. Cisplatin, fluorouracil, hydroxyurea, bleomycin, ifosfamide and their combination with other drugs are common medical choices with different pharmacological reactions, and the combination of chemo and radiotherapy could be further categorized into neoadjuvant, adjuvant and concurrent therapy due to whether the medication was performed before, after or concurrent with the process of radiation [13-14].

Fortunately, regardless of the administration time point, a large number of randomized control trials (RCTs) assessing the efficacy and safety between different chemotherapeutic agents after radiotherapy or radiotherapy alone had been carried out and provided with sufficient clinical data. However, some of them were contradicted to each other. Two trials appraised the concurrent radiation and cisplatin plus fluorouracil compared with radiation alone. Morris et al. reported a significant superiority in both overall survival (OS) and recurrence-free survival (RFS), but the same outcomes of Kim et al.'s trial were 1.03 and 1.01 without statistically significance. Meanwhile, although many meta-analyses (MA) existed, there was still a lack of a conclusive treatment strategy for patients with cervical cancer, and none of them conducted a network meta-analysis (NMA) among variety of chemotherapies to draw the best outcomes result in patients $[15,16]$.

As the first NMA on this subject, we synthesized both direct and indirect data to compare the efficacy among multiple common chemotherapies combined with radiotherapy in patients with cervical cancer after surgery. OS, RFS, the incidence of recurrence, and distant metastasis were used as the outcomes to assess cisplatin, cisplatin+fluorouracil, hydroxyurea, fluorouracil alone, cisplatin+ifosfamide, bleomycin + ifosfamide+cisplatin, mitomycin $\mathrm{C}+$ fluorouracil, and other 14 chemotherapeutic strategies. 5-year OS and PFS were considered as primary outcomes. This multiple-treatment Bayesian meta-analysis aimed to integrate the existed data and to draw a conclusion to offer a guideline for the corresponding clinical practice.

\section{RESULTS}

\section{Included studies}

This NMA included 39 eligible trials with 8,952 patients involved, screened from the 412 identified literatures from electronic database depending on the inclusion and exclusion criteria, which were published between March 1979 and October 2015 were included [13, 14, 17-53], and the efficacy of a total of 22 chemotherapies were evaluated. Besides, 113 meta-analysis or systematic reviews were retrieved through the keywords searching on the Internet. The characteristics of patients, the details of the specific interventions and the analyzed outcomes of each trial were listed in Table 1. Among 39 eligible trials, 34 of them gave the data of OS, 36 trials showed the PFS, 34 pairs of comparisons involved the data of recurrence, and the outcome of distant metastasis was analyzed in 24 trials. The directly compared connections among each chemotherapeutic agent for each outcome were displayed in Figure 1.

\section{Overall survival (OS)}

OS were treated as the primary outcomes. Since 1 -year OS was a short-term evaluation index showing no significant difference in most cases (Table 2), 3-year and 5-year OS were more reliable to reflect their efficacies. The hazard ratio (HR) of placebo was compared with 22 chemotherapies reported the HR of 3 -year OS. As shown in the lower panel of Table 3, cisplatin+ifosfamide+paclitaxel $(\mathrm{HR}=2.14,95 \% \mathrm{CrI}$ : 1.1-4.17), cisplatin+fluorouracil+hydroxyurea $(\mathrm{HR}=$ 1.78, 95\% CrI: 1.08- 2.93), cisplatin+paclitaxel $(\mathrm{HR}=$ 1.77, 95\% CrI: 1.23-2.54), fluorouracil +mitomycin C $(\mathrm{HR}=1.75,95 \% \mathrm{CrI}: 1.04-2.95)$, cisplatin+ifosfamide $(\mathrm{HR}=1.71,95 \% \mathrm{CrI}: 1.26-2.33)$, cisplatin+mitolactol $(\mathrm{HR}=1.57,95 \% \mathrm{CrI}: 1.11-2.23)$, cisplatin + topotecan $(\mathrm{HR}=0.69,95 \% \mathrm{CrI}: 0.50-0.96)$, cisplatin $(\mathrm{HR}=0.70$, 95\% CrI: 0.56-0.87), and cisplatin+fluorouracil (HR $=1.32,95 \% \mathrm{CrI}: 1.07-1.64)$, these nine interventions with $95 \% \mathrm{CrI}$ excluded 1.00 had dramatic advantage on improving the survival length of patients. Furthermore, for 5-year OS in the lower panel of Table 4, cisplatin+docetaxel $(\mathrm{HR}=2.22,95 \% \mathrm{CrI}: 1.27-3.87)$, ci splatin+fluorouracil+hydroxyurea $(\mathrm{HR}=1.81,95 \% \mathrm{CrI}$ : 1.15-2.86), fluorouracil+mitomycin $\mathrm{C}(\mathrm{HR}=1.67,95 \%$ CrI: $1.02-2.71)$, cisplatin $(\mathrm{HR}=1.44,95 \% \mathrm{CrI}: 1.15-$ $1.8)$, and cisplatin+fluorouracil $(\mathrm{HR}=1.29,95 \% \mathrm{CrI}$ : 1.05-1.59) had statistically significance compared with placebo. Results of ranking analysis in Table 5 suggested that cisplatin+fluorouracil+hydroxyurea was efficacious in 1-year and 5-year period. Another two favorable interventions in 5-year OS were cisplatin+docetaxel and fluorouracil+mitomycin $\mathrm{C}$, suggested a good therapeutic effect in long-term survival. 
Table 1: Baseline characteristics of included studies

\begin{tabular}{|c|c|c|c|c|c|c|c|c|c|c|c|c|c|c|}
\hline & Study & & & & Patient & & & & Intervention & & & & Outcome & \\
\hline Study & Country & RCT & $\begin{array}{l}\text { Follow- } \\
\text { up, m }\end{array}$ & $\mathbf{N}$ & $\begin{array}{l}\text { FIGO } \\
\text { stage }\end{array}$ & $\begin{array}{l}\text { Mean } \\
\text { age, } \mathbf{y}\end{array}$ & RT & Type & Contrast & $\begin{array}{l}\text { Dosage, } \\
\text { mg/m² or } \\
\text { else }\end{array}$ & OS & RFS & Recurrence & $\begin{array}{c}\text { Distant } \\
\text { metastasis }\end{array}$ \\
\hline Pu 2013 & China & $\sqrt{ }$ & 60 & $\begin{array}{l}140 \\
145\end{array}$ & IB-IIA & 45,47 & $\sqrt{ }$ & $\mathrm{c}$ & $\begin{array}{c}\text { Cis vs } \mathrm{Cis}+ \\
\text { Doc }\end{array}$ & $\begin{array}{c}40,30+ \\
30\end{array}$ & $\sqrt{ }$ & $\sqrt{ }$ & $\sqrt{ }$ & $\sqrt{ }$ \\
\hline Yin 2012 & China & - & 120 & $\begin{array}{l}148 \\
104\end{array}$ & IB2-IIB & NA & - & $\mathrm{n}$ & $\begin{array}{c}\text { Cis }+ \text { Pac vs } \\
\text { Nedaplatin }+ \\
\text { Pac }\end{array}$ & $\begin{array}{l}50+175 \\
50+175\end{array}$ & $\sqrt{ }$ & $\sqrt{ }$ & & \\
\hline $\begin{array}{l}\text { Symonds } \\
2000\end{array}$ & UK & $\sqrt{ }$ & 100 & $\begin{array}{l}104 \\
100\end{array}$ & IIB-IVA & 49,48 & $\sqrt{ }$ & $\mathrm{n}, \mathrm{r}$ & $\begin{array}{c}\text { Cis }+ \\
\text { Methotrexate } \\
\text { vs Placebo }\end{array}$ & $\begin{array}{l}50+ \\
100,-\end{array}$ & $\sqrt{ }$ & $\sqrt{ }$ & $\sqrt{ }$ & $\sqrt{ }$ \\
\hline Roberts 2000 & USA & $\sqrt{ }$ & 46 & $\begin{array}{l}78 \\
82\end{array}$ & IB2-IVA & $\begin{array}{l}47.5 \\
48.5\end{array}$ & $\sqrt{ }$ & $\mathrm{a}, \mathrm{r}$ & $\begin{array}{l}\text { MitoC vs } \\
\text { Placebo }\end{array}$ & $15,-$ & $\sqrt{ }$ & $\sqrt{ }$ & & \\
\hline Morris 1999 & USA & $\sqrt{ }$ & 60 & $\begin{array}{l}195 \\
193\end{array}$ & IB-IVA & 47,47 & $\sqrt{ }$ & $\mathrm{c}, \mathrm{r}$ & $\begin{array}{c}\text { Cis }+ \text { Flu vs } \\
\text { Placebo }\end{array}$ & $\begin{array}{c}75+ \\
4000,-\end{array}$ & $\sqrt{ }$ & $\sqrt{ }$ & $\sqrt{ }$ & \\
\hline Tseng 1997 & China & $\sqrt{ }$ & 60 & $\begin{array}{l}60 \\
62\end{array}$ & IIB/IIIB & NA & $\sqrt{ }$ & $\mathrm{c}, \mathrm{r}$ & $\begin{array}{c}\mathrm{Ble}+\mathrm{Cis}+\mathrm{Vcr} \\
\text { vs Placebo }\end{array}$ & $\begin{array}{c}25+50+ \\
1,-\end{array}$ & $\sqrt{ }$ & $\sqrt{ }$ & $\sqrt{ }$ & $\sqrt{ }$ \\
\hline Thomas 1998 & Canada & $\sqrt{ }$ & 80 & $\begin{array}{l}50 \\
49\end{array}$ & IB-IVA & NA & $\sqrt{ }$ & $\mathrm{c}, \mathrm{r}$ & Flu vs Placebo & $1000,-$ & & $\sqrt{ }$ & $\sqrt{ }$ & \\
\hline Sundfor 1996 & Norway & $\sqrt{ }$ & 60 & $\begin{array}{l}47 \\
47\end{array}$ & IIIB/IVA & $\begin{array}{l}52.7 \\
52.5\end{array}$ & $\sqrt{ }$ & $\mathrm{n}, \mathrm{r}$ & $\begin{array}{c}\text { Cis }+ \text { Flu vs } \\
\text { Placebo }\end{array}$ & $\begin{array}{c}100+ \\
1000,-\end{array}$ & $\sqrt{ }$ & $\sqrt{ }$ & $\sqrt{ }$ & $\sqrt{ }$ \\
\hline Rose 1999 & USA & $\sqrt{ }$ & 48 & $\begin{array}{l}176, \\
173, \\
177\end{array}$ & IIB-IVA & NA & $\sqrt{ }$ & $\mathrm{c}$ & $\begin{array}{c}\text { Cis vs Cis }+ \\
\text { Flu }+ \text { Hyd vs } \\
\text { Hyd }\end{array}$ & $\begin{array}{c}40,50+ \\
4000+ \\
2000 \\
3000\end{array}$ & $\sqrt{ }$ & $\sqrt{ }$ & $\sqrt{ }$ & \\
\hline Keys 1999 & USA & - & 48 & $\begin{array}{l}183 \\
186\end{array}$ & IB & NA & $\sqrt{ }$ & $\mathrm{c}, \mathrm{r}$ & Cis vs Placebo & $40,-$ & $\sqrt{ }$ & $\sqrt{ }$ & $\sqrt{ }$ & $\sqrt{ }$ \\
\hline Long 2005 & USA & $\sqrt{ }$ & 36 & $\begin{array}{l}147 \\
146\end{array}$ & IVB & 46,48 & $\sqrt{ }$ & $\mathrm{c}$ & $\begin{array}{c}\text { Cis }+ \\
\text { Topotecan vs } \\
\text { Cis }\end{array}$ & $\begin{array}{c}50+ \\
0.75,50\end{array}$ & $\sqrt{ }$ & $\sqrt{ }$ & $\sqrt{ }$ & \\
\hline Kim 2008 & Korea & $\sqrt{ }$ & 72 & $\begin{array}{l}78 \\
77\end{array}$ & IIB-IVA & 58,57 & $\sqrt{ }$ & $\mathrm{c}$ & $\begin{array}{c}\text { Cis }+ \text { Flu vs } \\
\text { Cis }\end{array}$ & $\begin{array}{c}20+ \\
1000,30\end{array}$ & $\sqrt{ }$ & $\sqrt{ }$ & $\sqrt{ }$ & $\sqrt{ }$ \\
\hline $\begin{array}{l}\text { Lanciano } \\
2005\end{array}$ & USA & $\sqrt{ }$ & 48 & $\begin{array}{l}159 \\
157\end{array}$ & IIB-IVA & NA & $\sqrt{ }$ & $\mathrm{c}$ & Cis vs Flu & 40,225 & $\sqrt{ }$ & $\sqrt{ }$ & $\sqrt{ }$ & $\sqrt{ }$ \\
\hline Peters 2000 & USA & $\sqrt{ }$ & 96 & $\begin{array}{l}127 \\
116\end{array}$ & IA2-IIA & 41,38 & $\sqrt{ }$ & $\mathrm{c}, \mathrm{r}$ & $\begin{array}{c}\text { Cis }+ \text { Flu vs } \\
\text { Placebo }\end{array}$ & $\begin{array}{c}70+ \\
1000,-\end{array}$ & $\sqrt{ }$ & $\sqrt{ }$ & $\sqrt{ }$ & $\sqrt{ }$ \\
\hline Whitney 1999 & USA & $\sqrt{ }$ & 72 & $\begin{array}{l}177 \\
191\end{array}$ & IIB-IVA & NA & $\sqrt{ }$ & $\mathrm{a}$ & $\begin{array}{c}\text { Cis }+ \text { Flu vs } \\
\text { Hyd }\end{array}$ & $\begin{array}{c}50+ \\
4000 \\
3000\end{array}$ & $\sqrt{ }$ & $\sqrt{ }$ & $\sqrt{ }$ & $\sqrt{ }$ \\
\hline Eifel 2004 & USA & $\sqrt{ }$ & 96 & $\begin{array}{l}195 \\
195\end{array}$ & IIB-IVA & NA & $\sqrt{ }$ & $\mathrm{c}, \mathrm{r}$ & $\begin{array}{c}\text { Cis }+ \text { Flu vs } \\
\text { Placebo }\end{array}$ & $\begin{array}{c}75+ \\
4000,-\end{array}$ & $\sqrt{ }$ & $\sqrt{ }$ & $\sqrt{ }$ & $\sqrt{ }$ \\
\hline Eddy 2007 & USA & $\sqrt{ }$ & 72 & $\begin{array}{l}145 \\
143\end{array}$ & IB & NA & $\sqrt{ }$ & $\mathrm{n}, \mathrm{r}$ & $\begin{array}{c}\text { Cis }+ \text { Ver vs } \\
\text { Placebo }\end{array}$ & $50+1,-$ & $\sqrt{ }$ & $\sqrt{ }$ & $\sqrt{ }$ & $\sqrt{ }$ \\
\hline Herod 2000 & UK & $\sqrt{ }$ & 84 & $\begin{array}{l}89 \\
88\end{array}$ & IB-IVA & 47,46 & $\sqrt{ }$ & $\mathrm{n}, \mathrm{r}$ & $\begin{array}{c}\text { Ble }+ \text { Cis }+ \text { Ifo } \\
\text { vs Placebo }\end{array}$ & $\begin{array}{c}30+50+ \\
5000,-\end{array}$ & $\sqrt{ }$ & & $\sqrt{ }$ & \\
\hline $\begin{array}{l}\text { Lorvidhaya } \\
2003\end{array}$ & Thailand & $\sqrt{ }$ & 120 & $\begin{array}{l}233 \\
221 \\
242\end{array}$ & IIB-IVA & $\begin{array}{c}48,49 \\
50\end{array}$ & $\sqrt{ }$ & $\begin{array}{l}\mathrm{c}, \\
\mathrm{a}, \mathrm{r}\end{array}$ & $\begin{array}{l}\text { Flu }+ \text { MitoC vs } \\
\text { Flu vs Placebo }\end{array}$ & $\begin{array}{c}300 \mathrm{mg} / \mathrm{d} \\
+10,200 \\
\mathrm{mg} / \mathrm{d},-\end{array}$ & $\sqrt{ }$ & $\sqrt{ }$ & $\sqrt{ }$ & $\sqrt{ }$ \\
\hline Buda 2005 & Italy & $\sqrt{ }$ & 60 & $\begin{array}{l}96 \\
108\end{array}$ & IB2-IVA & 47,49 & - & $\mathrm{n}$ & $\begin{array}{c}\text { Cis + Ifo + Pac } \\
\text { vs Cis + Ifo }\end{array}$ & $\begin{array}{c}75+ \\
5000+ \\
175,75+ \\
5000\end{array}$ & $\sqrt{ }$ & $\sqrt{ }$ & $\sqrt{ }$ & * \\
\hline
\end{tabular}

(Continued) 


\begin{tabular}{|c|c|c|c|c|c|c|c|c|c|c|c|c|c|c|}
\hline & Study & & & & Patient & & & & Intervention & & & & Outcome & \\
\hline Study & Country & RCT & $\begin{array}{l}\text { Follow- } \\
\text { up, m }\end{array}$ & $\mathbf{N}$ & $\begin{array}{l}\text { FIGO } \\
\text { stage }\end{array}$ & $\begin{array}{l}\text { Mean } \\
\text { age, } y\end{array}$ & RT & Type & Contrast & $\begin{array}{l}\text { Dosage, } \\
\mathrm{mg} / \mathrm{m}^{2} \text { or } \\
\text { else }\end{array}$ & OS & RFS & Recurrence & $\begin{array}{c}\text { Distant } \\
\text { metastasis }\end{array}$ \\
\hline $\begin{array}{l}\text { Leborgne } \\
1997\end{array}$ & Uruguay & $\sqrt{ }$ & 60 & $\begin{array}{l}48 \\
49\end{array}$ & IB-IVA & 47,43 & $\sqrt{ }$ & $\mathrm{a}, \mathrm{r}$ & $\begin{array}{c}\mathrm{Ble}+\mathrm{Cis}+\mathrm{Vcr} \\
\text { vs Placebo }\end{array}$ & $\begin{array}{c}25+50+ \\
1,-\end{array}$ & & $\sqrt{ }$ & $\sqrt{ }$ & \\
\hline Nedovic 2012 & Serbia & - & 42 & $\begin{array}{l}64 \\
70\end{array}$ & IIB-IVA & 51,54 & $\sqrt{ }$ & $\mathrm{c}$ & $\begin{array}{c}\text { Cis + Flu vs } \\
\text { Cis }\end{array}$ & $\begin{array}{c}75+ \\
4000,40\end{array}$ & $\sqrt{ }$ & $\sqrt{ }$ & $\sqrt{ }$ & $\sqrt{ }$ \\
\hline $\begin{array}{l}\text { Garipagaoglu } \\
2004\end{array}$ & Turkey & $\sqrt{ }$ & 60 & $\begin{array}{l}22 \\
22\end{array}$ & IIB/IIIB & $\begin{array}{l}50.5 \\
49.2\end{array}$ & $\sqrt{ }$ & $\mathrm{c}, \mathrm{r}$ & Cis vs Placebo & $20,-$ & $\sqrt{ }$ & $\sqrt{ }$ & $\sqrt{ }$ & $\sqrt{ }$ \\
\hline Moore 2004 & USA & - & 24 & $\begin{array}{l}130 \\
134\end{array}$ & IVB & $\begin{array}{c}48.5 \\
46\end{array}$ & - & $\mathrm{n}$ & $\begin{array}{c}\mathrm{Cis}+\mathrm{Pac} v \mathrm{~s} \\
\mathrm{Cis}\end{array}$ & $\begin{array}{c}50+135 \\
50\end{array}$ & $\sqrt{ }$ & $\sqrt{ }$ & & $\sqrt{ }$ \\
\hline Omura 1997 & USA & $\sqrt{ }$ & 24 & $\begin{array}{l}151 \\
147 \\
140\end{array}$ & IVB & $\begin{array}{l}46.3 \\
48.8 \\
47.3\end{array}$ & - & $\mathrm{n}$ & $\begin{array}{c}\text { Cis + Ifo vs Cis } \\
+ \text { Mitolactol } \\
\text { vs Cis }\end{array}$ & $\begin{array}{c}50+ \\
5000,50 \\
+180,50\end{array}$ & $\sqrt{ }$ & $\sqrt{ }$ & $\sqrt{ }$ & \\
\hline Pearcey 2002 & Canada & $\sqrt{ }$ & 120 & $\begin{array}{l}127 \\
126\end{array}$ & IIB-IVA & NA & $\sqrt{ }$ & $\mathrm{c}, \mathrm{r}$ & Cis vs Placebo & $40,-$ & $\sqrt{ }$ & $\sqrt{ }$ & $\sqrt{ }$ & $\sqrt{ }$ \\
\hline Bloss 2002 & USA & $\sqrt{ }$ & 24 & $\begin{array}{l}141 \\
146\end{array}$ & IVB & 46,45 & - & $\mathrm{n}$ & $\begin{array}{c}\text { Ble }+ \text { Cis + Ifo } \\
\text { vs Cis + Ifo }\end{array}$ & $\begin{array}{l}30 \text { units } \\
+50+ \\
5000,50 \\
+5000\end{array}$ & $\sqrt{ }$ & $\sqrt{ }$ & $\sqrt{ }$ & \\
\hline $\begin{array}{l}\text { Tattersall } \\
1992\end{array}$ & Australia & $\sqrt{ }$ & 75 & $\begin{array}{l}34 \\
37\end{array}$ & IIB-IVA & 54,56 & $\sqrt{ }$ & $\mathrm{c}, \mathrm{r}$ & $\begin{array}{c}\mathrm{Ble}+\mathrm{Cis}+\mathrm{Vbl} \\
\text { vs Placebo }\end{array}$ & $\begin{array}{c}15+50+ \\
4,-\end{array}$ & $\sqrt{ }$ & $\sqrt{ }$ & $\sqrt{ }$ & $\sqrt{ }$ \\
\hline $\begin{array}{l}\text { Tattersall } \\
1991\end{array}$ & Australia & $\sqrt{ }$ & 72 & $\begin{array}{l}34 \\
37\end{array}$ & IB-IIA & NA & $\sqrt{ }$ & $\mathrm{a}, \mathrm{r}$ & $\begin{array}{c}\mathrm{Ble}+\mathrm{Cis}+\mathrm{Vbl} \\
\text { vs Placebo }\end{array}$ & $\begin{array}{c}15+50+ \\
4,-\end{array}$ & & $\sqrt{ }$ & $\sqrt{ }$ & $\sqrt{ }$ \\
\hline $\begin{array}{l}\text { Tattersall } \\
1995\end{array}$ & Australia & $\sqrt{ }$ & 48 & $\begin{array}{l}129 \\
131\end{array}$ & IIB-IVA & 47,52 & $\sqrt{ }$ & $\mathrm{c}, \mathrm{r}$ & $\begin{array}{l}\text { Cis + Epi vs } \\
\text { Placebo }\end{array}$ & $\begin{array}{c}60+ \\
110,-\end{array}$ & $\sqrt{ }$ & $\sqrt{ }$ & $\sqrt{ }$ & $\sqrt{ }$ \\
\hline Wong 1988 & China & $\sqrt{ }$ & 70 & $\begin{array}{l}22 \\
25\end{array}$ & IIB-IIIB & NA & $\sqrt{ }$ & $\mathrm{c}, \mathrm{r}$ & Cis vs Placebo & $25,-$ & & $\sqrt{ }$ & $\sqrt{ }$ & $\sqrt{ }$ \\
\hline Wong 1999 & China & $\sqrt{ }$ & 140 & $\begin{array}{l}110 \\
110\end{array}$ & I-IIIB & $\begin{array}{l}52.4 \\
55.5\end{array}$ & $\sqrt{ }$ & $\mathrm{a}, \mathrm{r}$ & Epi vs Placebo & $90,-$ & $\sqrt{ }$ & $\sqrt{ }$ & $\sqrt{ }$ & $\sqrt{ }$ \\
\hline Tabata 2003 & Japan & $\sqrt{ }$ & 60 & $\begin{array}{l}32 \\
29\end{array}$ & IIIB/IVA & 57,59 & $\sqrt{ }$ & $\mathrm{a}, \mathrm{r}$ & $\begin{array}{c}\text { Ble }+ \text { Cis }+ \\
\text { MitoC + Vbl vs } \\
\text { Placebo }\end{array}$ & $\begin{array}{c}3+10+7 \\
+0.7\end{array}$ & $\sqrt{ }$ & & $\sqrt{ }$ & $\sqrt{ }$ \\
\hline Piver 1987 & USA & $\sqrt{ }$ & 156 & $\begin{array}{l}20 \\
25\end{array}$ & IIIB & NA & $\sqrt{ }$ & $\mathrm{c}, \mathrm{r}$ & Hyd vs Placebo & $\begin{array}{l}80 \mathrm{mg} / \\
\mathrm{kg},-\end{array}$ & $\sqrt{ }$ & $\sqrt{ }$ & $\sqrt{ }$ & \\
\hline Piver 1983 & USA & $\sqrt{ }$ & 110 & $\begin{array}{l}20 \\
20\end{array}$ & IIB & $\begin{array}{l}45.7 \\
50.5\end{array}$ & $\sqrt{ }$ & $\mathrm{c}, \mathrm{r}$ & Hyd vs Placebo & $\begin{array}{l}80 \mathrm{mg} / \\
\mathrm{kg},-\end{array}$ & $\sqrt{ }$ & & $\sqrt{ }$ & \\
\hline Nagai 2001 & Japan & $\sqrt{ }$ & 120 & $\begin{array}{l}32 \\
28\end{array}$ & II-IV & $\begin{array}{l}55.6 \\
64.4\end{array}$ & $\sqrt{ }$ & $\mathrm{a}$ & Cis vs Flu & $\begin{array}{c}120 \mathrm{mg} \\
200 \mathrm{mg} / \mathrm{d}\end{array}$ & $\sqrt{ }$ & $\sqrt{ }$ & & \\
\hline Kumar 1994 & India & $\sqrt{ }$ & 24 & $\begin{array}{l}94 \\
90\end{array}$ & IIB-IVA & $\begin{array}{l}45 \\
45.5\end{array}$ & $\sqrt{ }$ & $\mathrm{n}, \mathrm{r}$ & $\begin{array}{c}\text { Ble }+ \text { Cis }+ \text { Ifo } \\
\text { vs Placebo }\end{array}$ & $\begin{array}{c}15+50+ \\
1000,-\end{array}$ & & $\sqrt{ }$ & $\sqrt{ }$ & $\sqrt{ }$ \\
\hline $\begin{array}{l}\text { Hreshchyshyn } \\
1979\end{array}$ & USA & - & 48 & $\begin{array}{l}51 \\
46\end{array}$ & IIB-IVA & NA & $\sqrt{ }$ & $\mathrm{c}, \mathrm{r}$ & Hyd vs Placebo & $\begin{array}{l}80 \mathrm{mg} / \\
\mathrm{kg},-\end{array}$ & $\sqrt{ }$ & $\sqrt{ }$ & $\sqrt{ }$ & \\
\hline $\begin{array}{l}\text { Donnelly } \\
2015\end{array}$ & USA & - & 348 & $\begin{array}{l}42 \\
95 \\
99\end{array}$ & IB1-IVA & $\begin{array}{c}49 \\
(23- \\
83)\end{array}$ & $\sqrt{ }$ & $\begin{array}{l}\mathrm{c} \\
\mathrm{c}, \mathrm{r}\end{array}$ & $\begin{array}{l}\text { Cis }+ \text { Flu vs } \\
\text { Cis vs Placebo }\end{array}$ & $\begin{array}{c}70+ \\
1000, \\
40,-\end{array}$ & $\sqrt{ }$ & $\sqrt{ }$ & & \\
\hline
\end{tabular}

Note: FIGO, International Federation of Gynecology and Obstetrics; RT, radiationtherapy; OS, overall survival; RFS, recurrence-free survival; a, adjuvant; $\mathrm{n}$, neoadjuvant; c, concurrent; r, radiotherapy only.

*Data excluded to achieve network connectivity. 


\section{Recurrence-free survival (RFS)}

As to 3-year and 5-year RFS, another primary outcomes, were evaluated in 21 and 14 trials respectively. According to Tables 2-4, nine of them were noticeably better than treatments without chemotherapy for 3-year RFS, as mitomycin C ( $\mathrm{HR}=2,95 \% \mathrm{CI}: 1.11-3.61)$, cisplatin+ifosfamide+paclitaxel $(\mathrm{HR}=1.94,95 \% \mathrm{CrI}$ : 1.03-3.68), bleomycin+cisplatin+ifosfamide $(\mathrm{HR}=1.91$, 95\% CrI: $1.31-2.8)$, cisplatin+ifosfamide $(\mathrm{HR}=1.85,95 \%$ CrI: $1.33-2.56)$, cisplatin+paclitaxel $(\mathrm{HR}=1.64,95 \%$ CrI: 1.03-3.68), cisplatin+topotecan $(\mathrm{HR}=1.6,95 \% \mathrm{CrI}$ : 1.09-2.36), fluorouracil+mitomycin $\mathrm{C}(\mathrm{HR}=1.52,95 \%$ CrI: 1.04-2.21), cisplatin+fluorouracil $(\mathrm{HR}=1.45,95 \%$ CrI: 1.18-1.79), cisplatin ( $\mathrm{HR}=1.3,95 \%$ CrI: $1.05-1.6)$. Besides, mitomycin C (HR $=2.5,95 \%$ CI: 1.41-4.43), cisplatin+docetaxel (HR $=-2.01,95 \%$ CrI: $1.15-3.49)$, ci splatin+fluorouracil+hydroxyurea $(\mathrm{HR}=1.81,95 \% \mathrm{CrI}$ : 1.06-3.08), cisplatin+fluorouracil $(\mathrm{HR}=1.46,95 \% \mathrm{CrI}$ :
1.17-1.83), cisplatin $(\mathrm{HR}=1.29,95 \%$ CrI: $1.01-1.63)$ possessed obvious strength in 5 year RFS. In Table 5, ranking analysis showed that mitomycin $\mathrm{C}$ had excellent performance in both short term and long term RFS. Cisplatin+ifosfamide was also efficacious in 1-year and 3 -year RFS and since the lack of 5-year RFS, we could not estimate its long term efficacy. Cisplatin+docetaxel and cisplatin+ fluorouracil+hydroxyurea had advantage in improving 5-year RFS, which verified their outstanding efficacy in long-term RFS.

\section{Recurrence}

In terms of the secondary outcome of recurrence, 19 chemotherapy strategies were compared with placebo in Table 6. Cisplatin+ifosfamide+paclitaxel $(\mathrm{OR}=$ $0.15,95 \%$ CrI: $0.03-0.77)$, cisplatin+ ifosfamide $(\mathrm{OR}=$ 0.17, 95\% CrI: 0.05-0.53), cisplatin+fluorouracil (OR

B
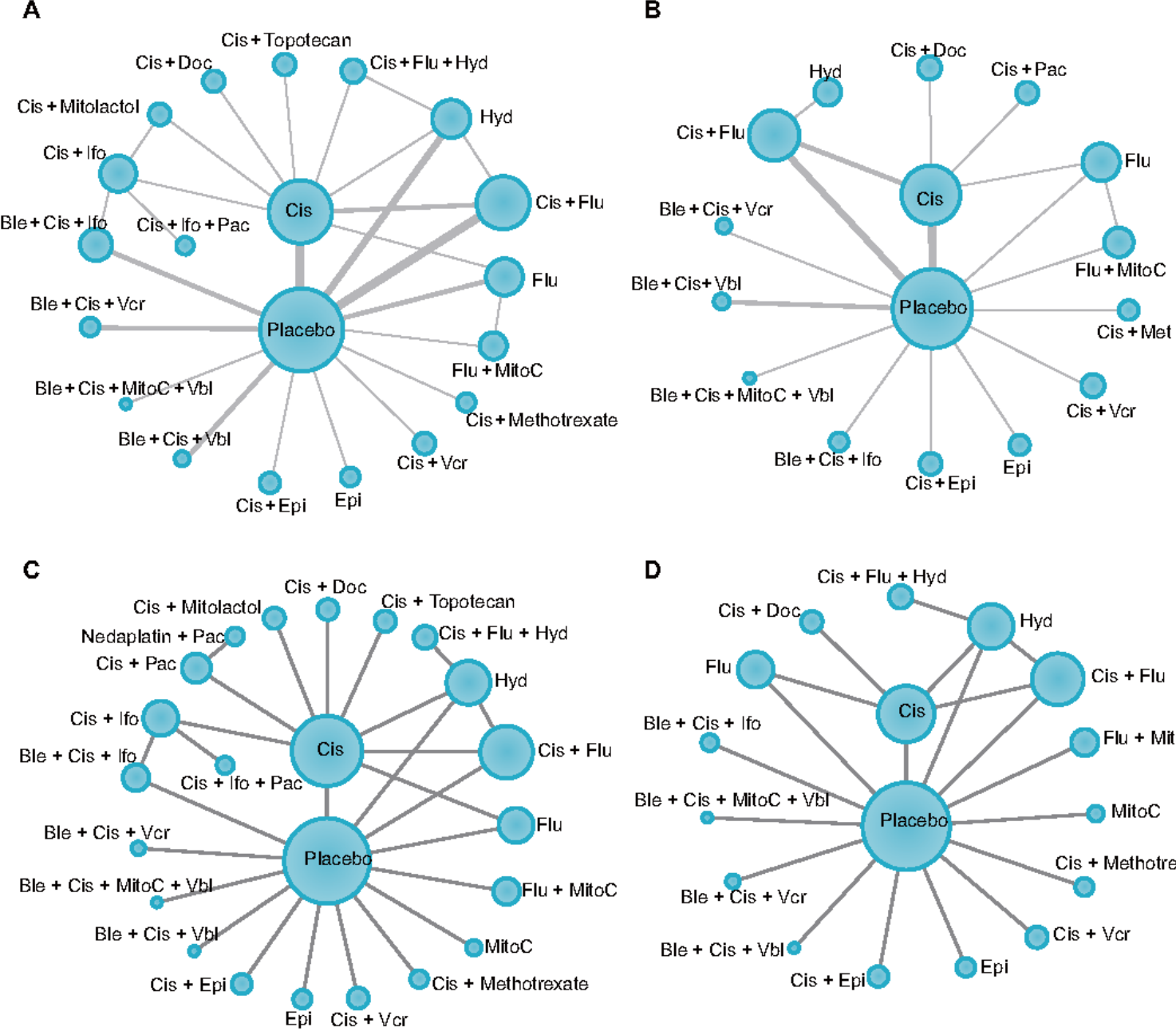

Figure 1: Network plots for each outcome. (A) recurrence; (B) distant metastasis; (C) 1-year and 3-year overall survival (OS) and recurrence-free survival (RFS); (D) 5-year OS and RFS. Each circle represents a specific treatment, and the size of the circles depends on the sample size involved in a specific treatment. The width of the line depends on the number of included studies for each comparison. OS, overall survival; RFS, recurrence-free survival. 
1-year RFS

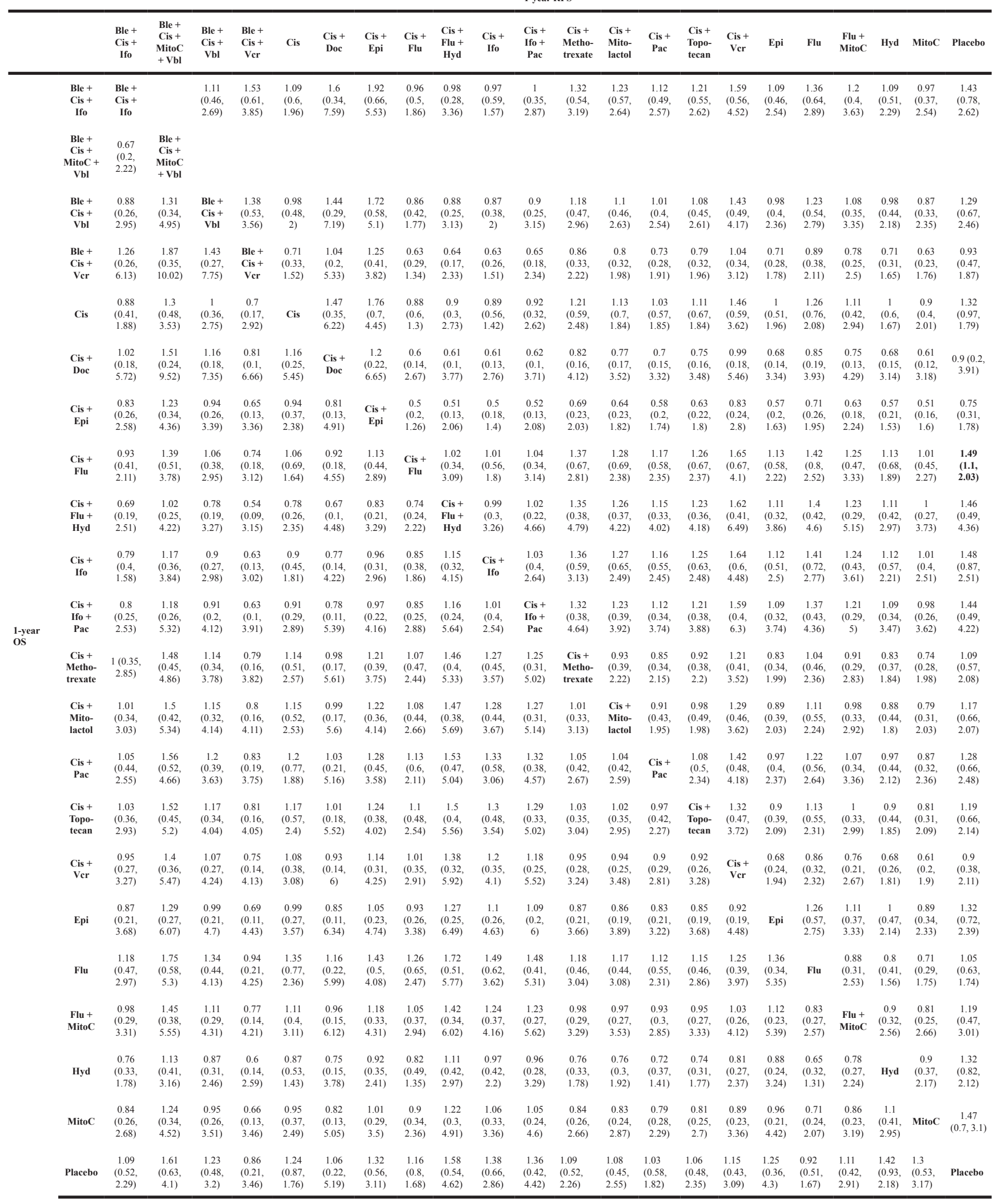




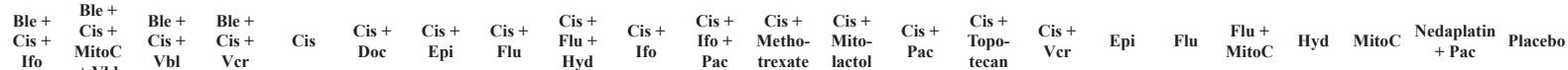
$\begin{array}{ccccccccccccccc}\begin{array}{c}\text { Cis } \\ \text { Ifo }\end{array} & \begin{array}{c}\text { MitoC } \\ + \text { Vbl }\end{array} & \text { Vbl } & \text { Ver } & \text { Doc } & \text { Epi } & \text { Flu } & \text { Hyd } & \text { Ifo } & \text { Pac } & \text { trexate lactol Pac } & \text { tecan } & \text { Ver } & \text { MitoC } & \end{array}$

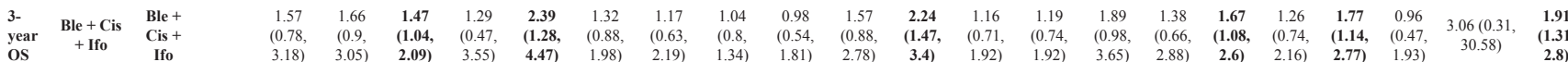
Ble + Cis $\quad 0.77 \quad$ Ble +

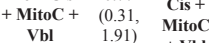

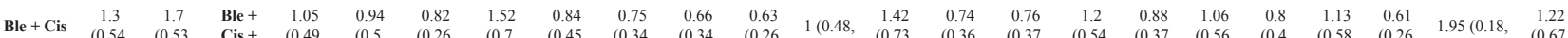

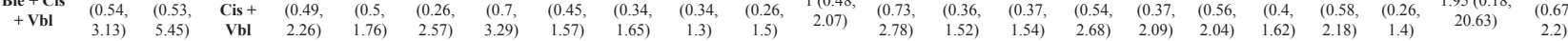

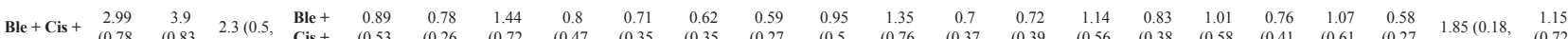

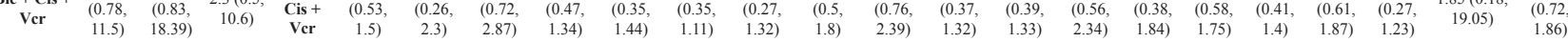
$\begin{array}{llllllllllllllllllllllll}\text { Cis } & 0.85 & 1.11 & 0.65 & 0.28 & & 0.88 & 1.62 & 0.9 & 0.8 & \mathbf{0 . 7} & 0.67 & 1.07 & 1.52 & 0.79 & 0.81 & 1.29 & 0.94 & 1.14 & 0.86 & 1.21 & 0.65 & 2.08(0.21, & \mathbf{1 . 3}\end{array}$

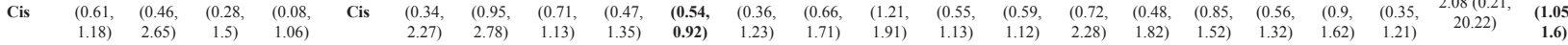

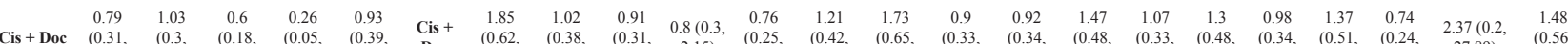

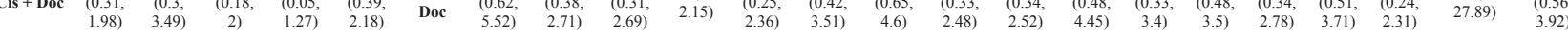

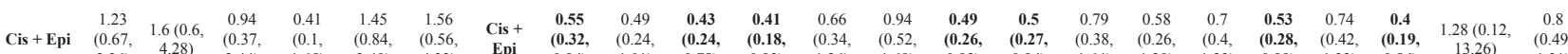

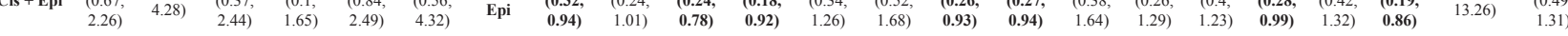

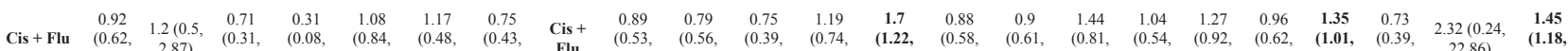

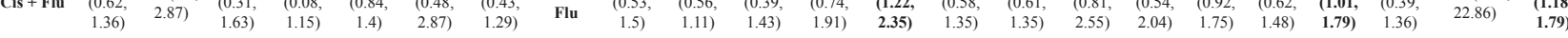

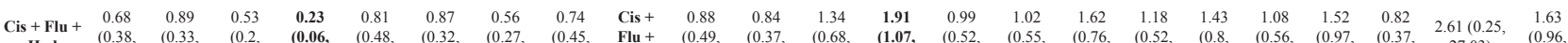

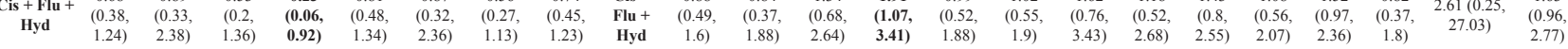

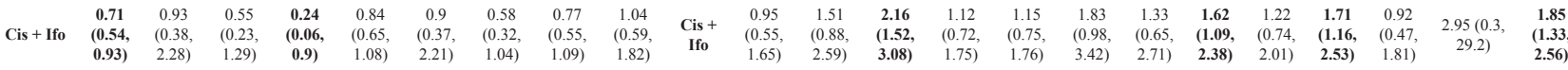

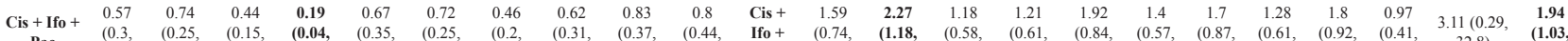

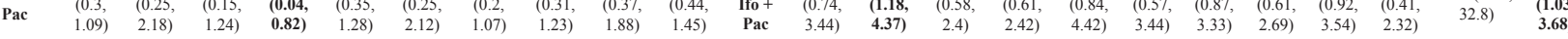

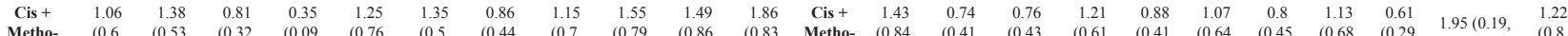

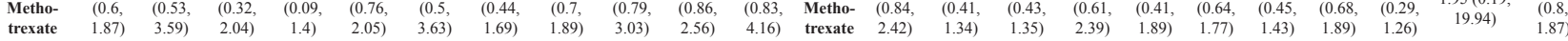

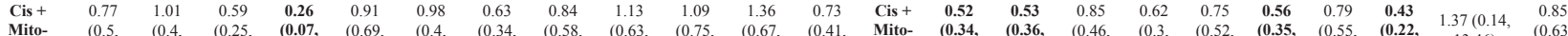
$\begin{array}{llllllllllllllllllllllll}\text { lactol } & 1.19) & 2.52) & 1.43) & \mathbf{0 . 9 9}) & 1.2) & 2.42) & 1.16) & 1.23) & 2.02) & 1.58) & 2.74) & 1.29) & \text { lactol } & \mathbf{0 . 7 9}) & \mathbf{0 . 7 9}) & 1.57) & 1.25) & 1.08) & \mathbf{0 . 9 2} & 1.15) & \mathbf{0 . 8 3} & 13.46) & 1.16)\end{array}$

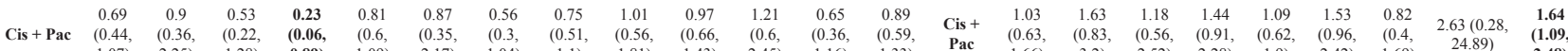

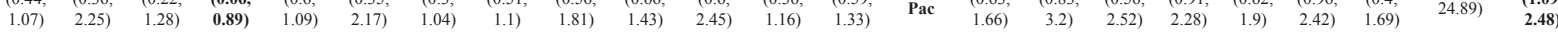

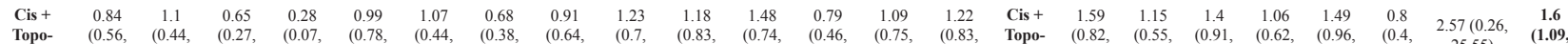

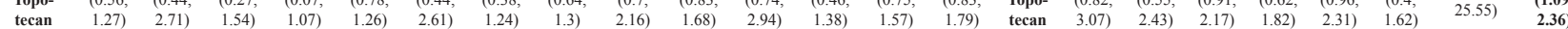

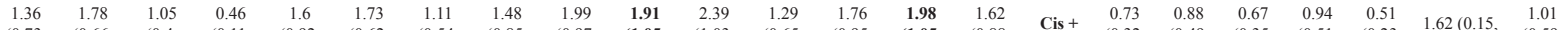

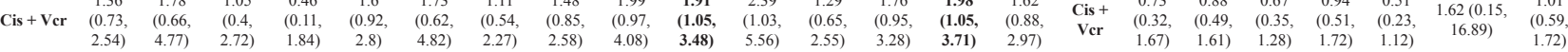
$\begin{array}{lllllllllllllllllllllll}1.05 & 1.37 & 0.8 & 0.35 & 1.23 & 1.33 & 0.85 & 1.14 & 1.53 & 1.47 & 1.84 & 0.99 & 1.35 & 1.52 & 1.24 & 0.77 & & 1.21 & 0.92 & 1.29 & 0.69 & 2.22(0.21, & 1.39\end{array}$

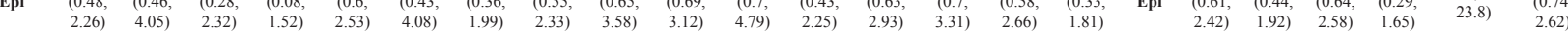

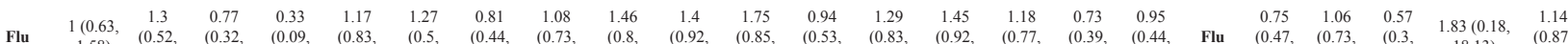

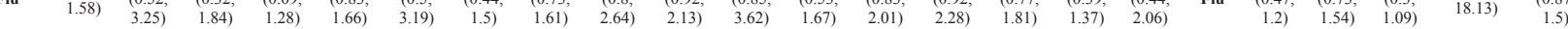

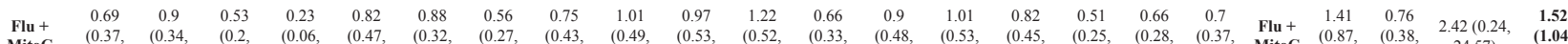

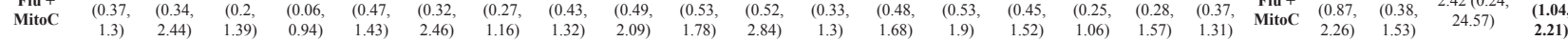
$\begin{array}{cccccccccccccccccccccccc} & 0.99 & 1.29 & 0.76 & 0.33 & 1.17 & 1.26 & 0.81 & 1.08 & 1.45 & 1.39 & 1.74 & 0.94 & 1.28 & 1.44 & 1.18 & 0.73 & 0.95 & 1(0.65, & 1.43 & & 0.54 & 1.72(0.17, & 1.08 \\ \text { Hyd } & (0.66, & (0.53, & (0.33, & (0.09, & (0.88, & (0.51, & (0.46, & (0.81, & (0.95, & (0.97, & (0.87, & (0.56, & (0.86, & (0.96, & (0.81, & (0.41, & (0.45, & 1.52) & (0.8, & \text { Hyd } & (0.28, & 1.72(7.1) & (0.81,\end{array}$

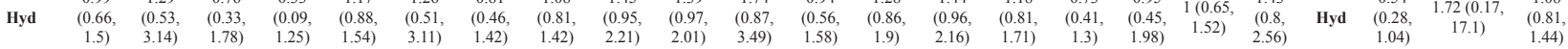

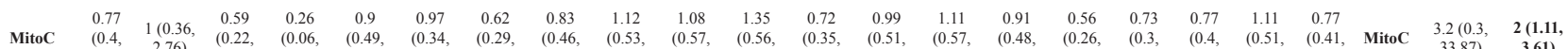
$\begin{array}{llllllllllllllllllllllll}1.49) & 2.76) & (0.22, & (0.06, & (0.49, & (0.34, & (0.29, & (0.46, & (0.53, & (0.57, & (0.56, & (0.35, & (0.51, & (0.57, & (0.48, & (0.26, & (0.3, & (0.4, & (0.51, & (0.41, & \text { MitoC } & 33.87) & 3.61)\end{array}$

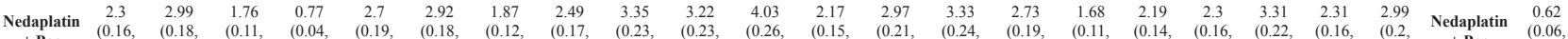

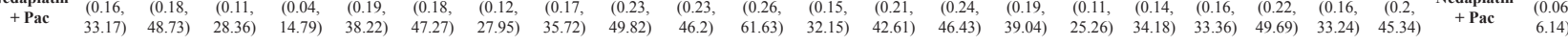

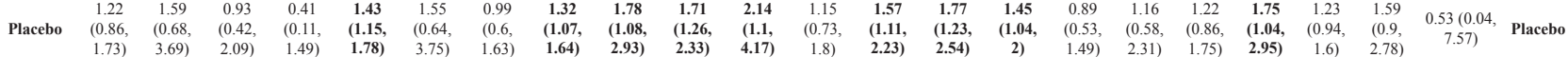




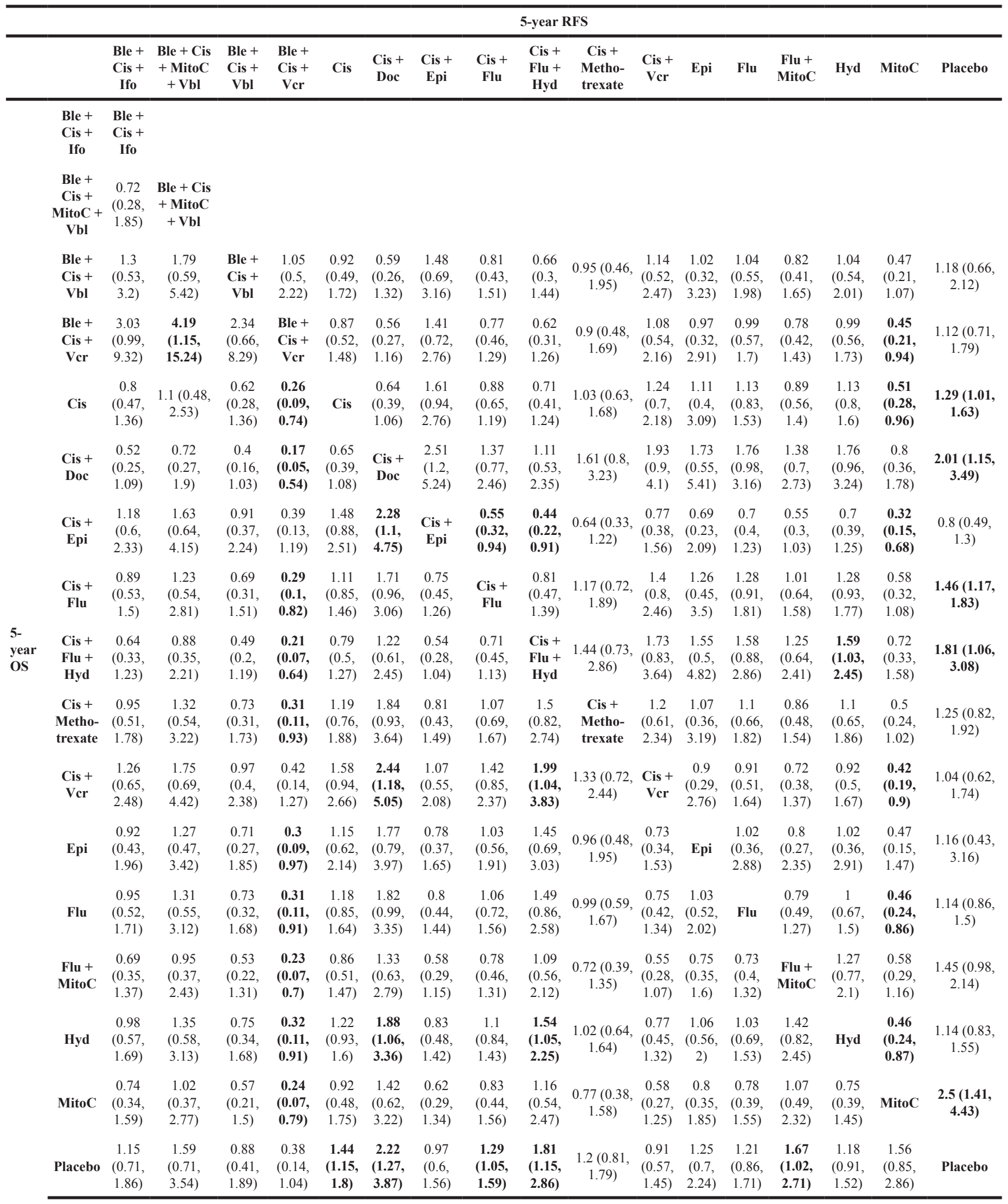


Table 5: $P$-score for each treatment under the outcomes of overall survival and recurrence-free survival

\begin{tabular}{|c|c|c|c|c|c|c|}
\hline \multirow{2}{*}{ Regimen } & \multicolumn{3}{|c|}{ Overall survival } & \multicolumn{3}{|c|}{ Recurrence-free survival } \\
\hline & $1 y$ & $3 y$ & $5 y$ & $1 y$ & $3 y$ & $5 y$ \\
\hline $\mathrm{Ble}+\mathrm{Cis}+\mathrm{Ifo}$ & 0.438 & 0.405 & 0.429 & 0.648 & 0.832 & - \\
\hline $\mathrm{Ble}+\mathrm{Cis}+\mathrm{MitoC}+\mathrm{Vbl}$ & 0.692 & 0.638 & 0.686 & - & - & - \\
\hline $\mathrm{Ble}+\mathrm{Cis}+\mathrm{Vbl}$ & 0.529 & 0.274 & 0.259 & 0.556 & 0.428 & 0.429 \\
\hline Ble + Cis + Ver & 0.348 & 0.070 & 0.024 & 0.307 & 0.374 & 0.373 \\
\hline Cis & 0.559 & 0.584 & 0.680 & 0.594 & 0.471 & 0.524 \\
\hline Cis + Doc & 0.453 & 0.618 & 0.918 & 0.359 & 0.576 & 0.857 \\
\hline Cis + Epi & 0.573 & 0.261 & 0.277 & 0.200 & 0.119 & 0.101 \\
\hline Cis + Flu & 0.493 & 0.494 & 0.555 & 0.716 & 0.598 & 0.674 \\
\hline Cis + Flu + Hyd & 0.670 & 0.768 & 0.834 & 0.623 & 0.688 & 0.801 \\
\hline Cis + Ifo & 0.619 & 0.771 & - & 0.690 & 0.810 & - \\
\hline Cis + Ifo + Pac & 0.582 & 0.856 & - & 0.612 & 0.799 & - \\
\hline Cis + Methotrexate & 0.442 & 0.373 & 0.474 & 0.417 & 0.422 & 0.483 \\
\hline Cis + Mitolactol & 0.438 & 0.685 & - & 0.466 & 0.118 & - \\
\hline Cis + Pac & 0.389 & 0.791 & - & 0.550 & 0.706 & - \\
\hline Cis + Topotecan & 0.423 & 0.593 & - & 0.483 & 0.686 & - \\
\hline Cis + Ver & 0.484 & 0.198 & 0.225 & 0.304 & 0.270 & 0.303 \\
\hline Epi & 0.531 & 0.406 & 0.511 & 0.577 & 0.541 & 0.430 \\
\hline Flu & 0.305 & 0.414 & 0.477 & 0.369 & 0.348 & 0.373 \\
\hline Flu + MitoC & 0.463 & 0.750 & 0.767 & 0.495 & 0.632 & 0.641 \\
\hline Hyd & 0.668 & 0.409 & 0.436 & 0.583 & 0.288 & 0.367 \\
\hline MitoC & 0.563 & 0.664 & 0.700 & 0.653 & 0.820 & 0.940 \\
\hline Nedaplatin + Pac & - & 0.264 & - & - & 0.264 & - \\
\hline Placebo & 0.338 & 0.214 & 0.248 & 0.296 & 0.212 & 0.203 \\
\hline
\end{tabular}

$=0.45,95 \%$ CrI: $0.29-0.71)$, fluorouracil $(\mathrm{OR}=0.51$, 95\% CrI: 0.26-0.96), and cisplatin (OR $=0.59,95 \%$ CrI: 0.37-0.93) were outstanding among them. Based on their SUCRA illustrated in Figure 2 and Table 8, cisplatin+ifosfamide (0.93) was the optimal combination, cisplatin+ifosfamide+paclitaxel (0.91) was the second, and the third one was cisplatin+docetaxel (0.74).

\section{Distant metastasis}

The potencies of reducing the occurrence of distant metastasis were also estimated, and 15 interventions had data in contrast with placebo, shown in Table 7. Only epirubicin (OR $=0.28,95 \%$ CrI: $0.08-0.91)$ was significantly excellent in reducing the occurrence of distant metastasis. This was also affirmed in our SUCRA rank probability diagram, seen in Figure 2 and Table 8 , since the SUCRA of epirubicin was 0.88 , and the following two were bleomycin+cisplatin+ifosfamide $(0.83)$, and cisplatin+docetaxel (0.73).

\section{Inconsistency test}

The heat plots in Figures 3-4 provided a detailed assessment of the inconsistency in this NMA. It appeared that there was no significant inconsistency identified within the net heat plot. Publication bias was visually using the comparison-adjusted funnel plots (Figure 5). We found no significant asymmetry patterns in funnel plots, so we concluded there were no significant publication bias in the included studies.

\section{DISCUSSION}

To appraise the efficacy of usual chemotherapies when combined with radiotherapy as concurrent, adjuvant, 


\begin{tabular}{|c|c|c|c|c|c|c|c|c|c|c|c|c|c|c|c|c|c|c|c|c|}
\hline & $\begin{array}{c}\mathrm{Ble}+\mathrm{Cis} \\
+ \text { Ifo }\end{array}$ & $\begin{array}{c}\text { Ble+Cis+ } \\
\text { MitoC+Vbl }\end{array}$ & $\begin{array}{c}\mathrm{Ble}+\mathrm{Cis} \\
+\mathrm{Vbl}\end{array}$ & $\begin{array}{c}\mathrm{Ble}+\mathrm{Cis} \\
+\mathrm{Vcr}\end{array}$ & Cis & Cis+Doc & Cis + Epi & Cis+Flu & Cis+Ifo & $\begin{array}{l}\text { Cis+Ifo } \\
+ \text { Pac }\end{array}$ & $\begin{array}{c}\text { Cis+ } \\
\text { Methotrexate }\end{array}$ & $\begin{array}{c}\text { Cis }+ \\
\text { Mitolactol }\end{array}$ & $\begin{array}{c}\text { Cist } \\
\text { Topotecan }\end{array}$ & Cis + Ver & Epi & Flu & $\begin{array}{c}\text { Cis+Flu } \\
+ \text { Hyd }\end{array}$ & $\begin{array}{c}\text { Flu+ } \\
\text { MitoC }\end{array}$ & Hyd & Placebo \\
\hline Ble+Cis+Ifo & $\begin{array}{c}\mathrm{Ble}^{+} \\
\text {Cis+Ifo }\end{array}$ & $\begin{array}{c}1.73(0.33 \\
8.40)\end{array}$ & $\begin{array}{c}1.68(0.48 \\
5.90)\end{array}$ & $\begin{array}{l}1.77 \\
(0.53 \\
5.58)\end{array}$ & $\begin{array}{c}0.85 \\
(0.33 \\
2.06)\end{array}$ & $\begin{array}{l}0.51 \\
(0.11, \\
2.12)\end{array}$ & $\begin{array}{c}1.72 \\
(0.44, \\
6.58)\end{array}$ & $\begin{array}{c}0.65 \\
(0.25, \\
1.59)\end{array}$ & $\begin{array}{c}0.24 \\
(0.07 \\
0.74)\end{array}$ & $\begin{array}{c}0.22(0.04 \\
1.09)\end{array}$ & $\begin{array}{c}1.18(0.23 \\
5.85)\end{array}$ & $\begin{array}{c}0.91(0.17 \\
5.27)\end{array}$ & $\begin{array}{c}0.61(0.13 \\
2.89)\end{array}$ & $\begin{array}{c}1.50(0.37 \\
5.48)\end{array}$ & $\begin{array}{c}0.77 \\
(0.18, \\
2.93)\end{array}$ & $\begin{array}{c}0.73 \\
(0.25 \\
2.00)\end{array}$ & $\begin{array}{l}0.62 \\
(0.16, \\
2.13)\end{array}$ & $\begin{array}{c}0.56 \\
(0.15 \\
1.92)\end{array}$ & $\begin{array}{l}1.02 \\
(0.33, \\
2.68)\end{array}$ & $\begin{array}{c}1.43 \\
(0.61, \\
3.20)\end{array}$ \\
\hline $\begin{array}{l}\text { Ble+Cis+ } \\
\text { MitoC+Vbl }\end{array}$ & $\begin{array}{c}0.58 \\
(0.12 \\
2.99)\end{array}$ & $\begin{array}{c}\mathrm{Ble+Cis} \\
+\mathrm{MitoC}+\mathrm{Vbl}\end{array}$ & $\begin{array}{l}0.98(0.19 \\
5.19)\end{array}$ & $\begin{array}{c}1.04 \\
(0.21 \\
5.24)\end{array}$ & $\begin{array}{c}0.49 \\
(0.11 \\
2.13)\end{array}$ & $\begin{array}{c}0.30 \\
(0.05, \\
1.81)\end{array}$ & $\begin{array}{l}1.01 \\
(0.17 \\
5.95)\end{array}$ & $\begin{array}{c}0.38 \\
(0.09 \\
1.61)\end{array}$ & $\begin{array}{c}0.14 \\
(0.02 \\
0.86)\end{array}$ & $\begin{array}{c}0.13(0.02 \\
1.08)\end{array}$ & $\begin{array}{c}0.69(0.10 \\
4.76)\end{array}$ & $\begin{array}{c}0.53(0.07 \\
5.08)\end{array}$ & $\begin{array}{c}0.36(0.05 \\
2.47)\end{array}$ & $\begin{array}{c}0.87(0.16 \\
4.95)\end{array}$ & $\begin{array}{c}0.44 \\
(0.08, \\
2.62)\end{array}$ & $\begin{array}{c}0.42 \\
(0.09 \\
1.93)\end{array}$ & $\begin{array}{c}0.36 \\
(0.07 \\
1.90)\end{array}$ & $\begin{array}{c}0.33 \\
(0.06 \\
1.74)\end{array}$ & $\begin{array}{l}0.59 \\
(0.13, \\
2.53)\end{array}$ & $\begin{array}{c}0.84 \\
(0.21, \\
3.32)\end{array}$ \\
\hline Ble + Cis + Vbl & $\begin{array}{c}0.60 \\
(0.17 \\
2.10)\end{array}$ & $\begin{array}{c}1.02(0.19 \\
5.34)\end{array}$ & $\begin{array}{c}\text { Ble+Cis } \\
+\mathrm{Vbl}\end{array}$ & $\begin{array}{c}1.06 \\
(0.29 \\
3.76)\end{array}$ & $\begin{array}{c}0.50 \\
(0.17 \\
1.46)\end{array}$ & $\begin{array}{c}0.30 \\
(0.06, \\
1.42)\end{array}$ & $\begin{array}{l}1.02 \\
(0.25, \\
4.33)\end{array}$ & $\begin{array}{c}0.38 \\
(0.13, \\
1.10)\end{array}$ & $\begin{array}{c}0.14 \\
(0.03 \\
0.65)\end{array}$ & $\begin{array}{c}0.13(0.02 \\
0.87)\end{array}$ & $\begin{array}{c}0.70(0.13 \\
3.75)\end{array}$ & $\begin{array}{c}0.54(0.09 \\
3.82)\end{array}$ & $\begin{array}{c}0.37(0.07 \\
1.85)\end{array}$ & $\begin{array}{c}0.89(0.20 \\
3.71)\end{array}$ & $\begin{array}{c}0.46 \\
(0.10 \\
1.99)\end{array}$ & $\begin{array}{c}0.43 \\
(0.13 \\
1.36)\end{array}$ & $\begin{array}{c}0.37 \\
(0.09, \\
1.44)\end{array}$ & $\begin{array}{l}0.33 \\
(0.08 \\
1.28)\end{array}$ & $\begin{array}{l}0.60 \\
(0.19, \\
1.78)\end{array}$ & $\begin{array}{c}0.85 \\
(0.33, \\
2.19)\end{array}$ \\
\hline Ble + Cis + Ver & $\begin{array}{c}0.56 \\
(0.18 \\
1.88)\end{array}$ & $\begin{array}{c}0.97(0.19 \\
4.83)\end{array}$ & $\begin{array}{c}0.95(0.27 \\
3.39)\end{array}$ & $\begin{array}{c}\text { Ble+Cis } \\
+ \text { Ver }\end{array}$ & $\begin{array}{c}0.48 \\
(0.18 \\
1.25)\end{array}$ & $\begin{array}{c}0.29 \\
(0.06 \\
1.27)\end{array}$ & $\begin{array}{c}0.97 \\
(0.25, \\
3.76)\end{array}$ & $\begin{array}{c}0.37 \\
(0.14 \\
0.94)\end{array}$ & $\begin{array}{c}0.14 \\
(0.03 \\
0.57)\end{array}$ & $\begin{array}{c}0.12(0.02 \\
0.78)\end{array}$ & $\begin{array}{c}0.67(0.13 \\
3.31)\end{array}$ & $\begin{array}{c}0.51(0.09 \\
3.56)\end{array}$ & $\begin{array}{c}0.34(0.07 \\
1.69)\end{array}$ & $\begin{array}{c}0.84(0.21 \\
3.27)\end{array}$ & $\begin{array}{c}0.43 \\
(0.10 \\
1.70)\end{array}$ & $\begin{array}{c}0.41 \\
(0.14 \\
1.17)\end{array}$ & $\begin{array}{c}0.35 \\
(0.09 \\
1.27)\end{array}$ & $\begin{array}{c}0.31 \\
(0.09 \\
1.12)\end{array}$ & $\begin{array}{c}0.57 \\
(0.19 \\
1.53)\end{array}$ & $\begin{array}{c}0.81 \\
(0.35, \\
1.87)\end{array}$ \\
\hline Cis & $\begin{array}{c}1.18 \\
(0.48 \\
3.05)\end{array}$ & $\begin{array}{c}2.03(0.47 \\
8.74)\end{array}$ & $\begin{array}{c}1.99(0.68 \\
5.73)\end{array}$ & $\begin{array}{c}2.10 \\
(0.80 \\
5.45)\end{array}$ & Cis & $\begin{array}{c}0.60 \\
(0.19 \\
1.86)\end{array}$ & $\begin{array}{c}2.05 \\
(0.64, \\
6.67)\end{array}$ & $\begin{array}{c}0.76 \\
(0.44, \\
1.31)\end{array}$ & $\begin{array}{c}0.29 \\
(0.09 \\
0.89)\end{array}$ & $\begin{array}{c}0.26(0.05 \\
1.31)\end{array}$ & $\begin{array}{c}1.40(0.32 \\
6.03)\end{array}$ & $\begin{array}{c}1.07(0.23, \\
6.03)\end{array}$ & $\begin{array}{c}0.73(0.21 \\
2.59)\end{array}$ & $\begin{array}{c}1.77(0.55 \\
5.67)\end{array}$ & $\begin{array}{c}0.91 \\
(0.27 \\
3.02)\end{array}$ & $\begin{array}{c}0.85 \\
(0.42 \\
1.71)\end{array}$ & $\begin{array}{c}0.74 \\
(0.27, \\
1.84)\end{array}$ & $\begin{array}{c}0.66 \\
(0.23 \\
1.89)\end{array}$ & $\begin{array}{l}1.21 \\
(0.59 \\
2.18)\end{array}$ & $\begin{array}{c}1.69 \\
(1.06, \\
2.68)\end{array}$ \\
\hline Cis+Doc & $\begin{array}{c}1.97 \\
(0.47 \\
8.71)\end{array}$ & $\begin{array}{c}3.38(0.55 \\
21.43)\end{array}$ & $\begin{array}{c}3.35(0.71 \\
16.05)\end{array}$ & $\begin{array}{c}3.51 \\
(0.79 \\
15.93)\end{array}$ & $\begin{array}{c}1.68 \\
(0.54 \\
5.27)\end{array}$ & Cis+Doc & $\begin{array}{c}3.39 \\
(0.66 \\
18.11)\end{array}$ & $\begin{array}{c}1.28 \\
(0.36 \\
4.55)\end{array}$ & $\begin{array}{c}0.48 \\
(0.10 \\
2.47)\end{array}$ & $\begin{array}{c}0.44(0.06 \\
3.23)\end{array}$ & $\begin{array}{c}2.34(0.37 \\
14.68)\end{array}$ & $\begin{array}{c}1.81(0.26 \\
14.25)\end{array}$ & $\begin{array}{c}1.22(0.22 \\
6.64)\end{array}$ & $\begin{array}{l}2.97(0.57 \\
15.12)\end{array}$ & $\begin{array}{c}1.52 \\
(0.29, \\
8.02)\end{array}$ & $\begin{array}{c}1.44 \\
(0.37 \\
5.51)\end{array}$ & $\begin{array}{l}1.23 \\
(0.26, \\
5.29)\end{array}$ & $\begin{array}{c}1.12 \\
(0.23 \\
5.28)\end{array}$ & $\begin{array}{l}2.01 \\
(0.50 \\
7.15)\end{array}$ & $\begin{array}{l}2.84 \\
(0.82 \\
9.70)\end{array}$ \\
\hline Cis + Epi & $\begin{array}{c}0.58 \\
(0.15 \\
2.27)\end{array}$ & $\begin{array}{c}0.99(0.17 \\
5.72)\end{array}$ & $\begin{array}{c}0.98(0.23 \\
4.08)\end{array}$ & $\begin{array}{c}1.03 \\
(0.27 \\
4.03)\end{array}$ & $\begin{array}{c}0.49 \\
(0.15 \\
1.55)\end{array}$ & $\begin{array}{c}0.29 \\
(0.06, \\
1.51)\end{array}$ & Cis + Epi & $\begin{array}{c}0.38 \\
(0.11 \\
1.19)\end{array}$ & $\begin{array}{c}0.14 \\
(0.03 \\
0.68)\end{array}$ & $\begin{array}{c}0.13(0.02 \\
0.91)\end{array}$ & $\begin{array}{c}0.68(0.12 \\
3.88)\end{array}$ & $\begin{array}{c}0.53(0.08 \\
3.96)\end{array}$ & $\begin{array}{c}0.36(0.06 \\
1.93)\end{array}$ & $\begin{array}{c}0.87(0.19 \\
3.85)\end{array}$ & $\begin{array}{c}0.45 \\
(0.09, \\
2.03)\end{array}$ & $\begin{array}{c}0.42 \\
(0.11 \\
1.45)\end{array}$ & $\begin{array}{c}0.36 \\
(0.08, \\
1.52)\end{array}$ & $\begin{array}{c}0.33 \\
(0.07 \\
1.36)\end{array}$ & $\begin{array}{c}0.59 \\
(0.16, \\
1.89)\end{array}$ & $\begin{array}{c}0.83 \\
(0.28 \\
2.38)\end{array}$ \\
\hline Cis+Flu & $\begin{array}{c}1.54 \\
(0.63 \\
4.03)\end{array}$ & $\begin{array}{c}2.65(0.62 \\
11.16)\end{array}$ & $\begin{array}{c}2.61(0.91 \\
7.46)\end{array}$ & $\begin{array}{c}2.73 \\
(1.07 \\
7.22)\end{array}$ & $\begin{array}{c}1.31 \\
(0.76 \\
2.26)\end{array}$ & $\begin{array}{c}0.78 \\
(0.22, \\
2.77)\end{array}$ & $\begin{array}{l}2.66 \\
(0.84, \\
8.77)\end{array}$ & Cis+Flu & $\begin{array}{c}0.38 \\
(0.11 \\
1.24)\end{array}$ & $\begin{array}{c}0.34(0.07 \\
1.83)\end{array}$ & $\begin{array}{c}1.83(0.42 \\
7.83)\end{array}$ & $\begin{array}{c}1.41(0.28 \\
8.31)\end{array}$ & $\begin{array}{c}0.95(0.24 \\
3.65)\end{array}$ & $\begin{array}{c}2.30(0.72 \\
7.41)\end{array}$ & $\begin{array}{c}1.18 \\
(0.36 \\
3.93)\end{array}$ & $\begin{array}{c}1.11 \\
(0.51 \\
2.43)\end{array}$ & $\begin{array}{c}0.96 \\
(0.33, \\
2.58)\end{array}$ & $\begin{array}{c}0.86 \\
(0.29 \\
2.49)\end{array}$ & $\begin{array}{l}1.57 \\
(0.77 \\
2.83)\end{array}$ & $\begin{array}{c}2.21 \\
(1.40 \\
3.53)\end{array}$ \\
\hline Cis+Ifo & $\begin{array}{c}4.08 \\
(1.35 \\
13.49)\end{array}$ & $\begin{array}{l}7.12(1.17 \\
42.43)\end{array}$ & $\begin{array}{c}6.91(1.55 \\
31.52)\end{array}$ & $\begin{array}{c}7.30 \\
(1.74 \\
30.66)\end{array}$ & $\begin{array}{r}3.47 \\
(1.13 \\
11.09)\end{array}$ & $\begin{array}{c}2.06 \\
(0.41 \\
10.46)\end{array}$ & $\begin{array}{c}7.10 \\
(1.48, \\
35.27)\end{array}$ & $\begin{array}{c}2.65 \\
(0.81 \\
9.06)\end{array}$ & CistIfo & $\begin{array}{c}0.90(0.30 \\
2.89)\end{array}$ & $\begin{array}{c}4.87(0.78 \\
30.22)\end{array}$ & $\begin{array}{c}3.71(0.92 \\
18.13)\end{array}$ & $\begin{array}{c}2.51(0.46 \\
13.93)\end{array}$ & $\begin{array}{l}6.09(1.28 \\
29.86)\end{array}$ & $\begin{array}{c}3.15 \\
(0.62, \\
15.87)\end{array}$ & $\begin{array}{c}2.96 \\
(0.81 \\
11.07)\end{array}$ & $\begin{array}{c}2.53 \\
(0.57 \\
10.86)\end{array}$ & $\begin{array}{c}2.29 \\
(0.51 \\
10.21)\end{array}$ & $\begin{array}{l}4.14 \\
(1.12, \\
14.63)\end{array}$ & $\begin{array}{c}5.86 \\
(1.90 \\
19.01)\end{array}$ \\
\hline Cis+Ifo+Pac & $\begin{array}{c}4.53 \\
(0.92 \\
22.95)\end{array}$ & $\begin{array}{c}7.72(0.93 \\
64.19)\end{array}$ & $\begin{array}{c}7.58(1.15 \\
48.69)\end{array}$ & $\begin{array}{c}8.00 \\
(1.27 \\
\mathbf{4 7 . 8 0 )}\end{array}$ & $\begin{array}{c}3.81 \\
(0.76 \\
18.89)\end{array}$ & $\begin{array}{c}2.28 \\
(0.31 \\
16.11)\end{array}$ & $\begin{array}{c}7.78 \\
(1.09 \\
54.27)\end{array}$ & $\begin{array}{l}2.92 \\
(0.55, \\
14.99)\end{array}$ & $\begin{array}{c}1.11 \\
(0.35 \\
3.31)\end{array}$ & $\begin{array}{c}\text { Cis+Ifo } \\
+ \text { Pac }\end{array}$ & $\begin{array}{c}5.36(0.61 \\
44.30)\end{array}$ & $\begin{array}{c}4.10(0.69, \\
27.85)\end{array}$ & $\begin{array}{c}2.75(0.35 \\
20.72)\end{array}$ & $\begin{array}{c}6.67(0.94 \\
47.95)\end{array}$ & $\begin{array}{c}3.43 \\
(0.48 \\
25.04)\end{array}$ & $\begin{array}{c}3.25 \\
(0.58 \\
18.35)\end{array}$ & $\begin{array}{c}2.79 \\
(0.43 \\
16.82)\end{array}$ & $\begin{array}{c}2.52 \\
(0.39 \\
16.28)\end{array}$ & $\begin{array}{l}4.61 \\
(0.79 \\
23.56)\end{array}$ & $\begin{array}{c}6.47 \\
(1.28 \\
31.85)\end{array}$ \\
\hline Cis+Methotrexate & $\begin{array}{c}0.85 \\
(0.17 \\
4.28)\end{array}$ & $\begin{array}{c}1.45(0.21 \\
10.09)\end{array}$ & $\begin{array}{c}1.42(0.27 \\
7.68)\end{array}$ & $\begin{array}{l}1.49 \\
(0.30 \\
7.58)\end{array}$ & $\begin{array}{c}0.72 \\
(0.17 \\
3.11)\end{array}$ & $\begin{array}{c}0.43 \\
(0.07, \\
2.67)\end{array}$ & $\begin{array}{c}1.46 \\
(0.26 \\
8.52)\end{array}$ & $\begin{array}{c}0.55 \\
(0.13, \\
2.36)\end{array}$ & $\begin{array}{c}0.21 \\
(0.03 \\
1.28)\end{array}$ & $\begin{array}{c}0.19(0.02 \\
1.63)\end{array}$ & $\begin{array}{l}\text { Cis+Metho- } \\
\text { trexate }\end{array}$ & $\begin{array}{c}0.78(0.10 \\
\quad 6.92)\end{array}$ & $\begin{array}{c}0.51(0.08 \\
3.57)\end{array}$ & $\begin{array}{c}1.26(0.22 \\
7.11)\end{array}$ & $\begin{array}{c}0.65 \\
(0.11, \\
3.91)\end{array}$ & $\begin{array}{c}0.61 \\
(0.13 \\
2.83)\end{array}$ & $\begin{array}{c}0.52 \\
(0.09, \\
2.79)\end{array}$ & $\begin{array}{c}0.47 \\
(0.09 \\
2.50)\end{array}$ & $\begin{array}{c}0.86 \\
(0.19 \\
3.69)\end{array}$ & $\begin{array}{l}1.21 \\
(0.31 \\
4.82)\end{array}$ \\
\hline Cis + Mitolactol & $\begin{array}{c}1.09 \\
(0.19 \\
5.90)\end{array}$ & $\begin{array}{c}1.90(0.20 \\
15.11)\end{array}$ & $\begin{array}{c}1.85(0.26 \\
11.69)\end{array}$ & $\begin{array}{c}1.97 \\
(0.28 \\
11.13)\end{array}$ & $\begin{array}{c}0.93 \\
(0.17 \\
4.32)\end{array}$ & $\begin{array}{c}0.55 \\
(0.07 \\
3.78)\end{array}$ & $\begin{array}{c}1.89 \\
(0.25, \\
12.86)\end{array}$ & $\begin{array}{c}0.71 \\
(0.12 \\
3.55)\end{array}$ & $\begin{array}{c}0.27 \\
(0.06 \\
1.09)\end{array}$ & $\begin{array}{c}0.24(0.04 \\
1.46)\end{array}$ & $\begin{array}{c}1.29(0.14 \\
10.22)\end{array}$ & $\begin{array}{l}\text { Cis+Mito- } \\
\text { lactol }\end{array}$ & $\begin{array}{c}0.67(0.08 \\
4.90)\end{array}$ & $\begin{array}{c}1.64(0.21, \\
10.64)\end{array}$ & $\begin{array}{c}0.84 \\
(0.10 \\
5.77)\end{array}$ & $\begin{array}{r}0.79 \\
(0.12 \\
4.18)\end{array}$ & $\begin{array}{c}0.68 \\
(0.09 \\
4.06)\end{array}$ & $\begin{array}{l}0.62 \\
(0.08 \\
3.85)\end{array}$ & $\begin{array}{l}1.11 \\
(0.17 \\
5.60)\end{array}$ & $\begin{array}{l}1.58 \\
(0.27 \\
7.52)\end{array}$ \\
\hline Cis+Topotecan & $\begin{array}{c}1.63 \\
(0.35 \\
7.93)\end{array}$ & $\begin{array}{c}2.79(0.41 \\
19.03)\end{array}$ & $\begin{array}{c}2.74(0.54 \\
14.35)\end{array}$ & $\begin{array}{c}2.90 \\
(0.59 \\
14.27)\end{array}$ & $\begin{array}{c}1.37 \\
(0.39 \\
4.81)\end{array}$ & $\begin{array}{c}0.82 \\
(0.15, \\
4.47)\end{array}$ & $\begin{array}{c}2.80 \\
(0.52, \\
15.94)\end{array}$ & $\begin{array}{c}1.05 \\
(0.27 \\
4.13)\end{array}$ & $\begin{array}{c}0.40 \\
(0.07 \\
2.18)\end{array}$ & $\begin{array}{c}0.36(0.05 \\
2.88)\end{array}$ & $\begin{array}{c}1.94(0.28 \\
12.66)\end{array}$ & $\begin{array}{c}1.50(0.20 \\
12.39)\end{array}$ & $\begin{array}{l}\text { Cis+Topo- } \\
\text { tecan }\end{array}$ & $\begin{array}{c}2.41(0.43 \\
13.44)\end{array}$ & $\begin{array}{c}1.25 \\
(0.22 \\
6.98)\end{array}$ & $\begin{array}{c}1.18 \\
(0.28 \\
4.94)\end{array}$ & $\begin{array}{l}1.01 \\
(0.20 \\
4.66)\end{array}$ & $\begin{array}{l}0.90 \\
(0.18 \\
4.57)\end{array}$ & $\begin{array}{c}1.65 \\
(0.39 \\
6.37)\end{array}$ & $\begin{array}{c}2.33 \\
(0.61, \\
8.94)\end{array}$ \\
\hline Cis + Ver & $\begin{array}{c}0.67 \\
(0.18 \\
2.70)\end{array}$ & $\begin{array}{c}1.16(0.20 \\
6.39)\end{array}$ & $\begin{array}{c}1.12(0.27 \\
4.91)\end{array}$ & $\begin{array}{c}1.19 \\
(0.31 \\
4.66)\end{array}$ & $\begin{array}{c}0.57 \\
(0.18 \\
1.82)\end{array}$ & $\begin{array}{c}0.34 \\
(0.07 \\
1.75)\end{array}$ & $\begin{array}{c}1.15 \\
(0.26 \\
5.20)\end{array}$ & $\begin{array}{c}0.43 \\
(0.13, \\
1.39)\end{array}$ & $\begin{array}{c}0.16 \\
(0.03 \\
0.78)\end{array}$ & $\begin{array}{c}0.15(0.02 \\
1.06)\end{array}$ & $\begin{array}{c}0.79(0.14 \\
4.46)\end{array}$ & $\begin{array}{c}0.61(0.09 \\
4.77)\end{array}$ & $\begin{array}{c}0.41(0.07 \\
2.32)\end{array}$ & CistVer & $\begin{array}{c}0.52 \\
(0.11, \\
2.40)\end{array}$ & $\begin{array}{c}0.49 \\
(0.14 \\
1.68)\end{array}$ & $\begin{array}{c}0.42 \\
(0.09 \\
1.74)\end{array}$ & $\begin{array}{c}0.38 \\
(0.09 \\
1.62)\end{array}$ & $\begin{array}{l}0.68 \\
(0.19 \\
2.18)\end{array}$ & $\begin{array}{c}0.96 \\
(0.33, \\
2.83)\end{array}$ \\
\hline Epi & $\begin{array}{c}1.30 \\
(0.34 \\
5.48)\end{array}$ & $\begin{array}{c}2.25(0.38 \\
13.27)\end{array}$ & $\begin{array}{c}2.19(0.50 \\
9.60)\end{array}$ & $\begin{array}{l}2.30 \\
(0.59 \\
9.59)\end{array}$ & $\begin{array}{c}1.10 \\
(0.33 \\
3.66)\end{array}$ & $\begin{array}{c}0.66 \\
(0.12 \\
3.45)\end{array}$ & $\begin{array}{c}2.24 \\
(0.49, \\
10.59)\end{array}$ & $\begin{array}{c}0.85 \\
(0.25, \\
2.78)\end{array}$ & $\begin{array}{c}0.32 \\
(0.06 \\
1.60)\end{array}$ & $\begin{array}{c}0.29(0.04 \\
2.07)\end{array}$ & $\begin{array}{c}1.54(0.26 \\
9.12)\end{array}$ & $\begin{array}{c}1.19(0.17, \\
9.58)\end{array}$ & $\begin{array}{c}0.80(0.14 \\
4.63)\end{array}$ & $\begin{array}{c}1.93(0.42 \\
9.12)\end{array}$ & Epi & $\begin{array}{c}0.94 \\
(0.26 \\
3.39)\end{array}$ & $\begin{array}{c}0.81 \\
(0.18 \\
3.45)\end{array}$ & $\begin{array}{c}0.72 \\
(0.16 \\
3.21)\end{array}$ & $\begin{array}{l}1.32 \\
(0.36, \\
4.47)\end{array}$ & $\begin{array}{c}1.87 \\
(0.62, \\
5.71)\end{array}$ \\
\hline Flu & $\begin{array}{c}1.37 \\
(0.50 \\
4.04)\end{array}$ & $\begin{array}{c}2.38(0.52 \\
11.00)\end{array}$ & $\begin{array}{c}2.34(0.73 \\
7.50)\end{array}$ & $\begin{array}{l}2.46 \\
(0.85 \\
7.23)\end{array}$ & $\begin{array}{c}1.17 \\
(0.59 \\
2.38)\end{array}$ & $\begin{array}{c}0.69 \\
(0.18 \\
2.71)\end{array}$ & $\begin{array}{c}2.38 \\
(0.69, \\
8.71)\end{array}$ & $\begin{array}{c}0.90 \\
(0.41, \\
1.96)\end{array}$ & $\begin{array}{c}0.34 \\
(0.09 \\
1.24)\end{array}$ & $\begin{array}{c}0.31(0.05 \\
1.73)\end{array}$ & $\begin{array}{c}1.64(0.35 \\
7.77)\end{array}$ & $\begin{array}{c}1.26(0.24 \\
8.06)\end{array}$ & $\begin{array}{c}0.85(0.20 \\
3.58)\end{array}$ & $\begin{array}{c}2.06(0.59 \\
7.20)\end{array}$ & $\begin{array}{c}1.06 \\
(0.30 \\
3.92)\end{array}$ & Flu & $\begin{array}{c}0.86 \\
(0.26 \\
2.69)\end{array}$ & $\begin{array}{l}0.77 \\
(0.29 \\
2.11)\end{array}$ & $\begin{array}{c}1.41 \\
(0.56 \\
3.16)\end{array}$ & $\begin{array}{c}1.98 \\
(1.03, \\
3.91)\end{array}$ \\
\hline Cis+Flu+Hyd & $\begin{array}{l}1.61 \\
(0.47 \\
6.28)\end{array}$ & $\begin{array}{c}2.79(0.53 \\
15.34)\end{array}$ & $\begin{array}{c}2.72(0.69 \\
11.11)\end{array}$ & $\begin{array}{l}2.85 \\
(0.79 \\
11.08)\end{array}$ & $\begin{array}{c}1.36 \\
(0.54 \\
3.74)\end{array}$ & $\begin{array}{c}0.81 \\
(0.19 \\
3.82)\end{array}$ & $\begin{array}{c}2.79 \\
(0.66 \\
12.37)\end{array}$ & $\begin{array}{c}1.04 \\
(0.39 \\
3.05)\end{array}$ & $\begin{array}{c}0.39 \\
(0.09 \\
1.76)\end{array}$ & $\begin{array}{c}0.36(0.06 \\
2.34)\end{array}$ & $\begin{array}{c}1.91(0.36 \\
10.73)\end{array}$ & $\begin{array}{c}1.47(0.25 \\
10.55)\end{array}$ & $\begin{array}{c}0.99(0.21 \\
5.00)\end{array}$ & $\begin{array}{c}2.39(0.57 \\
10.76)\end{array}$ & $\begin{array}{c}1.24 \\
(0.29 \\
5.68)\end{array}$ & $\begin{array}{c}1.16 \\
(0.37 \\
3.81)\end{array}$ & $\begin{array}{c}\text { Cis+Flu } \\
+ \text { Hyd }\end{array}$ & $\begin{array}{c}0.90 \\
(0.24 \\
3.75)\end{array}$ & $\begin{array}{l}1.64 \\
(0.61, \\
4.13)\end{array}$ & $\begin{array}{c}2.30 \\
(0.88 \\
6.50)\end{array}$ \\
\hline Flu+MitoC & $\begin{array}{l}1.78 \\
(0.52, \\
6.68)\end{array}$ & $\begin{array}{c}3.07(0.57, \\
16.69)\end{array}$ & $\begin{array}{c}3.02(0.78 \\
11.79)\end{array}$ & $\begin{array}{c}3.18 \\
(0.90 \\
11.66)\end{array}$ & $\begin{array}{c}1.51 \\
(0.53 \\
4.27)\end{array}$ & $\begin{array}{c}0.90 \\
(0.19 \\
4.32)\end{array}$ & $\begin{array}{c}3.06 \\
(0.74 \\
13.35)\end{array}$ & $\begin{array}{l}1.16 \\
(0.40 \\
3.43)\end{array}$ & $\begin{array}{c}0.44 \\
(0.10 \\
1.95)\end{array}$ & $\begin{array}{c}0.40(0.06 \\
2.58)\end{array}$ & $\begin{array}{c}2.11(0.40, \\
11.57)\end{array}$ & $\begin{array}{c}1.62(0.26 \\
11.85)\end{array}$ & $\begin{array}{c}1.11(0.22 \\
5.51)\end{array}$ & $\begin{array}{c}2.66(0.62 \\
11.38)\end{array}$ & $\begin{array}{c}1.38 \\
(0.31 \\
6.10)\end{array}$ & $\begin{array}{c}1.29 \\
(0.47 \\
3.44)\end{array}$ & $\begin{array}{l}1.11 \\
(0.27, \\
4.24)\end{array}$ & $\begin{array}{c}\text { Flut } \\
\text { MitoC }\end{array}$ & $\begin{array}{c}1.82 \\
(0.56 \\
5.36)\end{array}$ & $\begin{array}{l}2.56 \\
(0.95, \\
6.80)\end{array}$ \\
\hline Hyd & $\begin{array}{c}0.98 \\
(0.37 \\
3.02)\end{array}$ & $\begin{array}{c}1.69(0.40, \\
7.94)\end{array}$ & $\begin{array}{c}1.67(0.56 \\
5.31)\end{array}$ & $\begin{array}{l}1.75 \\
(0.66 \\
5.30)\end{array}$ & $\begin{array}{c}0.83 \\
(0.46 \\
1.70)\end{array}$ & $\begin{array}{c}0.50 \\
(0.14 \\
2.00)\end{array}$ & $\begin{array}{c}1.69 \\
(0.53, \\
6.32)\end{array}$ & $\begin{array}{c}0.64 \\
(0.35, \\
1.29)\end{array}$ & $\begin{array}{c}0.24 \\
(0.07 \\
0.89)\end{array}$ & $\begin{array}{c}0.22(0.04 \\
1.27)\end{array}$ & $\begin{array}{c}1.16(0.27, \\
5.40)\end{array}$ & $\begin{array}{c}0.90(0.18 \\
5.81)\end{array}$ & $\begin{array}{c}0.61(0.16 \\
2.58)\end{array}$ & $\begin{array}{c}1.46(0.46 \\
5.29)\end{array}$ & $\begin{array}{c}0.76 \\
(0.22, \\
2.80)\end{array}$ & $\begin{array}{c}0.71 \\
(0.32 \\
1.79)\end{array}$ & $\begin{array}{c}0.61 \\
(0.24, \\
1.63)\end{array}$ & $\begin{array}{c}0.55 \\
(0.19 \\
1.79)\end{array}$ & Hyd & $\begin{array}{c}1.41 \\
(0.81, \\
2.73)\end{array}$ \\
\hline Placebo & $\begin{array}{c}0.70 \\
(0.31 \\
1.64)\end{array}$ & $\begin{array}{c}1.19(0.30 \\
4.73)\end{array}$ & $\begin{array}{c}1.18(0.46 \\
3.05)\end{array}$ & $\begin{array}{l}1.24 \\
(0.54 \\
2.85)\end{array}$ & $\begin{array}{c}0.59 \\
(0.37 \\
0.94)\end{array}$ & $\begin{array}{c}0.35 \\
(0.10 \\
1.23)\end{array}$ & $\begin{array}{c}1.20 \\
(0.42, \\
3.60)\end{array}$ & $\begin{array}{c}0.45 \\
(0.28 \\
0.71)\end{array}$ & $\begin{array}{c}0.17 \\
(0.05 \\
0.53)\end{array}$ & $\begin{array}{c}0.15(0.03 \\
0.78)\end{array}$ & $\begin{array}{c}0.83(0.21 \\
3.27)\end{array}$ & $\begin{array}{c}0.63(0.13 \\
3.66)\end{array}$ & $\begin{array}{c}0.43(0.11 \\
1.64)\end{array}$ & $\begin{array}{c}1.04(0.35 \\
3.03)\end{array}$ & $\begin{array}{c}0.54 \\
(0.18, \\
1.61)\end{array}$ & $\begin{array}{c}0.51 \\
(0.26 \\
0.97)\end{array}$ & $\begin{array}{c}0.43 \\
(0.15, \\
1.13)\end{array}$ & $\begin{array}{c}0.39 \\
(0.15 \\
1.05)\end{array}$ & $\begin{array}{c}0.71 \\
(0.37 \\
1.24)\end{array}$ & Placebo \\
\hline
\end{tabular}

or neoadjuvant treatment to patients with cervical cancer after surgery, $39 \mathrm{RCTs}$ or clinical trials covering 22 interventions were analyzed, in terms of OS, RFS, the incidence of recurrence, and distant metastasis, in this NMA. It is the first issue in this domain, which integrated both the direct evidence and the indirect comparison remedying the insufficiency of the traditional metaanalysis.
In accordance with results, the combination of two or three medicines with diverse functional mechanisms had better impact than mono-chemotherapy. On the basis of biochemical mechanisms, relevant agents can be classified into four types, drugs affecting the structure and function of DNA, drugs interfering protein synthesis and function, drugs intercalating DNA to interfere the transcription as well as drugs used for metabolism inhibition.Statistically, 
Table 7: Mixed evidence of distant metastasis for different treatments

\begin{tabular}{|c|c|c|c|c|c|c|c|c|c|c|c|c|c|c|c|c|}
\hline & $\begin{array}{c}\text { Ble }+ \text { Cis } \\
\quad+\text { Ifo }\end{array}$ & $\begin{array}{c}\text { Ble+Cis+ } \\
\text { MitoC }+ \text { Vbl }\end{array}$ & $\begin{array}{c}\text { Ble }+ \text { Cis } \\
+ \text { Vbl }\end{array}$ & $\begin{array}{c}\text { Ble }+ \text { Cis } \\
+ \text { Ver }\end{array}$ & Cis & Cis +Doc & Cis+Epi & Cis + Flu & $\begin{array}{l}\text { Cis }+ \text { Metho- } \\
\text { trexate }\end{array}$ & Cis+Pac & Cis + Ver & Epi & Flu & $\begin{array}{c}\text { Flu+ } \\
\text { MitoC }\end{array}$ & Hyd & Placebo \\
\hline $\mathrm{Ble}+\mathrm{Cis}+\mathrm{Ifo}$ & $\begin{array}{c}\text { Ble+Cis } \\
\quad+\text { Ifo }\end{array}$ & $\begin{array}{c}5.29(0.65 \\
51.92)\end{array}$ & $\begin{array}{c}2.19 \\
(0.38 \\
14.21)\end{array}$ & $\begin{array}{l}2.22 \\
(0.34 \\
16.32)\end{array}$ & $\begin{array}{c}2.75 \\
(0.59 \\
15.69)\end{array}$ & $\begin{array}{c}1.42 \\
(0.15 \\
14.00)\end{array}$ & $\begin{array}{c}2.43 \\
(0.40 \\
17.76)\end{array}$ & $\begin{array}{l}2.50 \\
(0.55 \\
14.39)\end{array}$ & $\begin{array}{c}4.02(0.66 \\
28.87)\end{array}$ & $\begin{array}{c}4.04 \\
(0.65 \\
29.51)\end{array}$ & $\begin{array}{l}2.68 \\
(0.45 \\
18.96)\end{array}$ & $\begin{array}{c}0.92 \\
(0.14 \\
6.74)\end{array}$ & $\begin{array}{l}2.03 \\
(0.40, \\
12.01)\end{array}$ & $\begin{array}{c}2.02 \\
(0.36 \\
12.74)\end{array}$ & $\begin{array}{c}2.69 \\
(0.43, \\
22.55)\end{array}$ & $\begin{array}{l}3.29 \\
(0.79 \\
16.69)\end{array}$ \\
\hline $\begin{array}{l}\text { Ble+Cis } \\
+\mathrm{MitoC}+\mathrm{Vbl}\end{array}$ & $\begin{array}{c}0.19 \\
(0.02 \\
1.53)\end{array}$ & $\begin{array}{c}\text { Ble+Cis + } \\
\text { MitoC+Vbl }\end{array}$ & $\begin{array}{c}0.41 \\
(0.06 \\
2.64)\end{array}$ & $\begin{array}{c}0.42 \\
(0.05 \\
2.88)\end{array}$ & $\begin{array}{c}0.52 \\
(0.09 \\
2.70)\end{array}$ & $\begin{array}{c}0.26 \\
(0.02 \\
2.58)\end{array}$ & $\begin{array}{c}0.46 \\
(0.06 \\
3.16)\end{array}$ & $\begin{array}{c}0.47 \\
(0.09 \\
2.57)\end{array}$ & $\begin{array}{c}0.77(0.10 \\
5.07)\end{array}$ & $\begin{array}{c}0.76 \\
(0.10 \\
5.47)\end{array}$ & $\begin{array}{c}0.50 \\
(0.07 \\
3.30)\end{array}$ & $\begin{array}{c}0.18 \\
(0.02 \\
1.28)\end{array}$ & $\begin{array}{c}0.38 \\
(0.06 \\
2.14)\end{array}$ & $\begin{array}{c}0.38 \\
(0.06 \\
2.39)\end{array}$ & $\begin{array}{c}0.50 \\
(0.07 \\
3.95)\end{array}$ & $\begin{array}{c}0.63 \\
(0.12 \\
3.03)\end{array}$ \\
\hline $\mathrm{Ble}+\mathrm{Cis}+\mathrm{Vbl}$ & $\begin{array}{c}0.46 \\
(0.07 \\
2.65)\end{array}$ & $\begin{array}{c}2.46(0.38 \\
17.09)\end{array}$ & $\begin{array}{c}\text { Ble }+ \text { Cis } \\
+ \text { Vbl }\end{array}$ & $\begin{array}{l}1.02 \\
(0.21 \\
5.11)\end{array}$ & $\begin{array}{c}1.29 \\
(0.37 \\
4.25)\end{array}$ & $\begin{array}{l}0.67 \\
(0.09 \\
4.65)\end{array}$ & $\begin{array}{c}1.15 \\
(0.25 \\
5.13)\end{array}$ & $\begin{array}{c}1.17 \\
(0.36 \\
4.06)\end{array}$ & $\begin{array}{c}1.92(0.40 \\
8.31)\end{array}$ & $\begin{array}{c}1.90 \\
(0.38, \\
8.67)\end{array}$ & $\begin{array}{c}1.25 \\
(0.27 \\
5.50)\end{array}$ & $\begin{array}{c}0.44 \\
(0.08 \\
2.07)\end{array}$ & $\begin{array}{r}0.95 \\
(0.25 \\
3.35)\end{array}$ & $\begin{array}{c}0.94 \\
(0.22 \\
3.78)\end{array}$ & $\begin{array}{c}1.24 \\
(0.25 \\
7.02)\end{array}$ & $\begin{array}{l}1.54 \\
(0.53 \\
4.32)\end{array}$ \\
\hline $\mathrm{Ble}+\mathrm{Cis}+\mathrm{Vcr}$ & $\begin{array}{c}0.45 \\
(0.06 \\
2.97)\end{array}$ & $\begin{array}{c}2.38(0.35 \\
18.82)\end{array}$ & $\begin{array}{c}0.98 \\
(0.20 \\
4.79)\end{array}$ & $\begin{array}{c}\mathrm{Ble}+\mathrm{Cis} \\
+\mathrm{Vcr}\end{array}$ & $\begin{array}{r}1.24 \\
(0.32 \\
4.73)\end{array}$ & $\begin{array}{c}0.65 \\
(0.08, \\
4.98)\end{array}$ & $\begin{array}{c}1.10 \\
(0.21 \\
5.91)\end{array}$ & $\begin{array}{l}1.12 \\
(0.31 \\
4.80)\end{array}$ & $\begin{array}{c}1.87(0.36 \\
9.48)\end{array}$ & $\begin{array}{c}1.81 \\
(0.33 \\
9.93)\end{array}$ & $\begin{array}{c}1.21 \\
(0.23 \\
6.07)\end{array}$ & $\begin{array}{c}0.42 \\
(0.08 \\
2.27)\end{array}$ & $\begin{array}{c}0.92 \\
(0.21 \\
3.68)\end{array}$ & $\begin{array}{c}0.92 \\
(0.19 \\
4.26)\end{array}$ & $\begin{array}{r}1.21 \\
(0.23 \\
7.69)\end{array}$ & $\begin{array}{l}1.50 \\
(0.45 \\
4.99)\end{array}$ \\
\hline Cis & $\begin{array}{c}0.36 \\
(0.06 \\
1.68)\end{array}$ & $\begin{array}{c}1.92(0.37 \\
11.15)\end{array}$ & $\begin{array}{c}0.77 \\
(0.24 \\
2.70)\end{array}$ & $\begin{array}{c}0.80 \\
(0.21 \\
3.10)\end{array}$ & Cis & $\begin{array}{c}0.51 \\
(0.10, \\
2.46)\end{array}$ & $\begin{array}{c}0.89 \\
(0.25 \\
3.24)\end{array}$ & $\begin{array}{c}0.90 \\
(0.48 \\
1.92)\end{array}$ & $\begin{array}{c}1.48(0.42 \\
5.18)\end{array}$ & $\begin{array}{l}1.48 \\
(0.52, \\
4.15)\end{array}$ & $\begin{array}{c}0.98 \\
(0.27 \\
3.29)\end{array}$ & $\begin{array}{c}0.34 \\
(0.09 \\
1.27)\end{array}$ & $\begin{array}{c}0.74 \\
(0.33 \\
1.57)\end{array}$ & $\begin{array}{c}0.74 \\
(0.25 \\
2.11)\end{array}$ & $\begin{array}{c}0.96 \\
(0.28 \\
3.88)\end{array}$ & $\begin{array}{l}1.20 \\
(0.65 \\
2.16)\end{array}$ \\
\hline Cis+Doc & $\begin{array}{c}0.70 \\
(0.07 \\
6.59)\end{array}$ & $\begin{array}{c}3.81(0.39 \\
40.21)\end{array}$ & $\begin{array}{c}1.48 \\
(0.22, \\
11.65)\end{array}$ & $\begin{array}{c}1.53 \\
(0.20, \\
12.84)\end{array}$ & $\begin{array}{l}1.95 \\
(0.41, \\
10.07)\end{array}$ & Cis+Doc & $\begin{array}{c}1.72 \\
(0.23 \\
13.70)\end{array}$ & $\begin{array}{l}1.75 \\
(0.34 \\
11.17)\end{array}$ & $\begin{array}{c}2.84(0.38 \\
23.43)\end{array}$ & $\begin{array}{l}2.82 \\
(0.44, \\
19.85)\end{array}$ & $\begin{array}{l}1.90 \\
(0.25 \\
14.22)\end{array}$ & $\begin{array}{c}0.66 \\
(0.08 \\
5.24)\end{array}$ & $\begin{array}{c}1.43 \\
(0.24 \\
8.61)\end{array}$ & $\begin{array}{c}1.42 \\
(0.21 \\
9.99)\end{array}$ & $\begin{array}{l}1.88 \\
(0.27 \\
16.27)\end{array}$ & $\begin{array}{l}2.33 \\
(0.43, \\
13.23)\end{array}$ \\
\hline Cis+Epi & $\begin{array}{c}0.41 \\
(0.06 \\
2.53)\end{array}$ & $\begin{array}{c}2.19(0.32 \\
16.23)\end{array}$ & $\begin{array}{c}0.87 \\
(0.20 \\
4.08)\end{array}$ & $\begin{array}{c}0.91 \\
(0.17 \\
4.67)\end{array}$ & $\begin{array}{c}1.13 \\
(0.31 \\
4.02)\end{array}$ & $\begin{array}{c}0.58 \\
(0.07 \\
4.32)\end{array}$ & Cis+Epi & $\begin{array}{c}1.02 \\
(0.30 \\
3.89)\end{array}$ & $\begin{array}{c}1.66(0.33 \\
8.04)\end{array}$ & $\begin{array}{c}1.66 \\
(0.31 \\
8.47)\end{array}$ & $\begin{array}{c}1.09 \\
(0.22 \\
5.01)\end{array}$ & $\begin{array}{c}0.39 \\
(0.07 \\
1.95)\end{array}$ & $\begin{array}{c}0.83 \\
(0.20 \\
3.24)\end{array}$ & $\begin{array}{c}0.83 \\
(0.18 \\
3.53)\end{array}$ & $\begin{array}{c}1.09 \\
(0.22 \\
6.59)\end{array}$ & $\begin{array}{l}1.35 \\
(0.43 \\
4.14)\end{array}$ \\
\hline Cis+Flu & $\begin{array}{c}0.40 \\
(0.07 \\
1.80)\end{array}$ & $\begin{array}{c}2.13(0.39 \\
11.76)\end{array}$ & $\begin{array}{c}0.86 \\
(0.25 \\
2.75)\end{array}$ & $\begin{array}{c}0.89 \\
(0.21 \\
3.21)\end{array}$ & $\begin{array}{c}1.11 \\
(0.52 \\
2.08)\end{array}$ & $\begin{array}{c}0.57 \\
(0.09 \\
2.97)\end{array}$ & $\begin{array}{c}0.98 \\
(0.26 \\
3.35)\end{array}$ & Cis + Flu & $\begin{array}{c}1.65(0.43, \\
5.40)\end{array}$ & $\begin{array}{c}1.64 \\
(0.43, \\
5.31)\end{array}$ & $\begin{array}{c}1.09 \\
(0.28 \\
3.38)\end{array}$ & $\begin{array}{c}0.37 \\
(0.09 \\
1.36)\end{array}$ & $\begin{array}{c}0.82 \\
(0.29 \\
1.84)\end{array}$ & $\begin{array}{c}0.83 \\
(0.24 \\
2.29)\end{array}$ & $\begin{array}{c}1.07 \\
(0.35 \\
3.31)\end{array}$ & $\begin{array}{l}1.33 \\
(0.68 \\
2.23)\end{array}$ \\
\hline $\begin{array}{l}\text { Cis+Metho- } \\
\text { trexate }\end{array}$ & $\begin{array}{c}0.25 \\
(0.03 \\
1.52)\end{array}$ & $\begin{array}{c}1.30(0.20 \\
9.64)\end{array}$ & $\begin{array}{c}0.52 \\
(0.12 \\
2.50)\end{array}$ & $\begin{array}{c}0.54 \\
(0.11 \\
2.80)\end{array}$ & $\begin{array}{c}0.67 \\
(0.19 \\
2.37)\end{array}$ & $\begin{array}{c}0.35 \\
(0.04 \\
2.63)\end{array}$ & $\begin{array}{c}0.60 \\
(0.12, \\
3.05)\end{array}$ & $\begin{array}{c}0.61 \\
(0.19 \\
2.33)\end{array}$ & $\begin{array}{l}\text { Cis }+ \text { Metho- } \\
\text { trexate }\end{array}$ & $\begin{array}{c}1.00 \\
(0.20 \\
5.27)\end{array}$ & $\begin{array}{c}0.66 \\
(0.13, \\
3.12)\end{array}$ & $\begin{array}{c}0.23 \\
(0.04 \\
1.18)\end{array}$ & $\begin{array}{c}0.50 \\
(0.13, \\
1.93)\end{array}$ & $\begin{array}{c}0.50 \\
(0.11 \\
2.14)\end{array}$ & $\begin{array}{c}0.65 \\
(0.13, \\
3.88)\end{array}$ & $\begin{array}{c}0.82 \\
(0.27 \\
2.47)\end{array}$ \\
\hline Cis + Pac & $\begin{array}{c}0.25 \\
(0.03 \\
1.54)\end{array}$ & $\begin{array}{c}1.32(0.18 \\
10.06)\end{array}$ & $\begin{array}{c}0.53 \\
(0.12 \\
2.62)\end{array}$ & $\begin{array}{c}0.55 \\
(0.10 \\
3.00)\end{array}$ & $\begin{array}{c}0.68 \\
(0.24 \\
1.92)\end{array}$ & $\begin{array}{c}0.35 \\
(0.05 \\
2.28)\end{array}$ & $\begin{array}{c}0.60 \\
(0.12, \\
3.19)\end{array}$ & $\begin{array}{c}0.61 \\
(0.19 \\
2.31)\end{array}$ & $\begin{array}{c}1.00(0.19 \\
5.12)\end{array}$ & Cis + Pac & $\begin{array}{c}0.66 \\
(0.13 \\
3.25)\end{array}$ & $\begin{array}{c}0.23 \\
(0.04 \\
1.24)\end{array}$ & $\begin{array}{c}0.50 \\
(0.13, \\
1.83)\end{array}$ & $\begin{array}{c}0.50 \\
(0.11 \\
2.16)\end{array}$ & $\begin{array}{c}0.65 \\
(0.14 \\
3.76)\end{array}$ & $\begin{array}{c}0.82 \\
(0.24 \\
2.66)\end{array}$ \\
\hline Cis + Ver & $\begin{array}{c}0.37 \\
(0.05 \\
2.23)\end{array}$ & $\begin{array}{c}1.98(0.30 \\
14.48)\end{array}$ & $\begin{array}{c}0.80 \\
(0.18 \\
3.75)\end{array}$ & $\begin{array}{c}0.82 \\
(0.16 \\
4.30)\end{array}$ & $\begin{array}{c}1.02 \\
(0.30 \\
3.72)\end{array}$ & $\begin{array}{c}0.53 \\
(0.07 \\
4.00)\end{array}$ & $\begin{array}{c}0.92 \\
(0.20 \\
4.59)\end{array}$ & $\begin{array}{c}0.92 \\
(0.30 \\
3.52)\end{array}$ & $\begin{array}{c}1.51(0.32 \\
7.42)\end{array}$ & $\begin{array}{c}1.52 \\
(0.31 \\
7.63)\end{array}$ & Cis + Ver & $\begin{array}{c}0.35 \\
(0.07 \\
1.77)\end{array}$ & $\begin{array}{c}0.76 \\
(0.20 \\
3.01)\end{array}$ & $\begin{array}{c}0.76 \\
(0.18 \\
3.36)\end{array}$ & $\begin{array}{c}1.00 \\
(0.21 \\
6.02)\end{array}$ & $\begin{array}{c}1.24 \\
(0.42 \\
3.75)\end{array}$ \\
\hline Epi & $\begin{array}{l}1.08 \\
(0.15 \\
7.01)\end{array}$ & $\begin{array}{c}5.65(0.78 \\
44.07)\end{array}$ & $\begin{array}{l}2.29 \\
(0.48 \\
11.93)\end{array}$ & $\begin{array}{c}2.36 \\
(0.44 \\
13.23)\end{array}$ & $\begin{array}{l}2.93 \\
(0.79 \\
11.66)\end{array}$ & $\begin{array}{c}1.51 \\
(0.19 \\
12.41)\end{array}$ & $\begin{array}{c}2.59 \\
(0.51 \\
14.69)\end{array}$ & $\begin{array}{l}2.67 \\
(0.73, \\
11.15)\end{array}$ & $\begin{array}{c}4.36(0.85 \\
22.93)\end{array}$ & $\begin{array}{c}4.29 \\
(0.81 \\
24.10)\end{array}$ & $\begin{array}{c}2.89 \\
(0.56 \\
14.54)\end{array}$ & Epi & $\begin{array}{c}2.16 \\
(0.52 \\
9.33)\end{array}$ & $\begin{array}{r}2.15 \\
(0.47 \\
10.39)\end{array}$ & $\begin{array}{l}2.83 \\
(0.57 \\
18.09)\end{array}$ & $\begin{array}{c}3.55 \\
(1.09 \\
12.39)\end{array}$ \\
\hline Flu & $\begin{array}{c}0.49 \\
(0.08 \\
2.51)\end{array}$ & $\begin{array}{c}2.61(0.47 \\
16.14)\end{array}$ & $\begin{array}{c}1.06 \\
(0.30 \\
4.07)\end{array}$ & $\begin{array}{l}1.09 \\
(0.27 \\
4.68)\end{array}$ & $\begin{array}{c}1.36 \\
(0.64 \\
3.06)\end{array}$ & $\begin{array}{c}0.70 \\
(0.12, \\
4.12)\end{array}$ & $\begin{array}{c}1.21 \\
(0.31 \\
5.04)\end{array}$ & $\begin{array}{c}1.22 \\
(0.54 \\
3.46)\end{array}$ & $\begin{array}{c}2.01(0.52 \\
7.82)\end{array}$ & $\begin{array}{c}2.00 \\
(0.55 \\
7.60)\end{array}$ & $\begin{array}{c}1.32 \\
(0.33 \\
5.09)\end{array}$ & $\begin{array}{c}0.46 \\
(0.11 \\
1.94)\end{array}$ & Flu & $\begin{array}{c}1.00 \\
(0.37 \\
2.70)\end{array}$ & $\begin{array}{r}1.29 \\
(0.35 \\
6.33)\end{array}$ & $\begin{array}{c}1.63 \\
(0.76 \\
3.66)\end{array}$ \\
\hline Flu+MitoC & $\begin{array}{c}0.49 \\
(0.08 \\
2.81)\end{array}$ & $\begin{array}{c}2.62(0.42 \\
17.61)\end{array}$ & $\begin{array}{l}1.06 \\
(0.26 \\
4.63)\end{array}$ & $\begin{array}{c}1.09 \\
(0.23 \\
5.20)\end{array}$ & $\begin{array}{c}1.35 \\
(0.47 \\
3.99)\end{array}$ & $\begin{array}{c}0.70 \\
(0.10 \\
4.70)\end{array}$ & $\begin{array}{c}1.21 \\
(0.28 \\
5.56)\end{array}$ & $\begin{array}{l}1.20 \\
(0.44, \\
4.12)\end{array}$ & $\begin{array}{c}1.99(0.47 \\
8.80)\end{array}$ & $\begin{array}{c}2.00 \\
(0.46 \\
9.11)\end{array}$ & $\begin{array}{c}1.32 \\
(0.30 \\
5.52)\end{array}$ & $\begin{array}{c}0.46 \\
(0.10 \\
2.15)\end{array}$ & $\begin{array}{l}1.00 \\
(0.37 \\
2.70)\end{array}$ & $\begin{array}{c}\text { Flu+ } \\
\text { MitoC }\end{array}$ & $\begin{array}{c}1.31 \\
(0.30 \\
7.13)\end{array}$ & $\begin{array}{l}1.62 \\
(0.63 \\
4.33)\end{array}$ \\
\hline Hyd & $\begin{array}{c}0.37 \\
(0.04 \\
2.33)\end{array}$ & $\begin{array}{c}2.02(0.25 \\
14.72)\end{array}$ & $\begin{array}{c}0.81 \\
(0.14, \\
3.99)\end{array}$ & $\begin{array}{c}0.83 \\
(0.13, \\
4.42)\end{array}$ & $\begin{array}{c}1.04 \\
(0.26 \\
3.52)\end{array}$ & $\begin{array}{c}0.53 \\
(0.06 \\
3.70)\end{array}$ & $\begin{array}{c}0.91 \\
(0.15 \\
4.54)\end{array}$ & $\begin{array}{c}0.93 \\
(0.30 \\
2.83)\end{array}$ & $\begin{array}{c}1.54(0.26 \\
7.51)\end{array}$ & $\begin{array}{c}1.53 \\
(0.27 \\
7.27)\end{array}$ & $\begin{array}{c}1.00 \\
(0.17 \\
4.77)\end{array}$ & $\begin{array}{c}0.35 \\
(0.06 \\
1.77)\end{array}$ & $\begin{array}{c}0.77 \\
(0.16 \\
2.87)\end{array}$ & $\begin{array}{c}0.76 \\
(0.14 \\
3.30)\end{array}$ & Hyd & $\begin{array}{l}1.24 \\
(0.32 \\
3.95)\end{array}$ \\
\hline Placebo & $\begin{array}{c}0.30 \\
(0.06 \\
1.26)\end{array}$ & $\begin{array}{c}1.59(0.33 \\
8.26)\end{array}$ & $\begin{array}{c}0.65 \\
(0.23 \\
1.89)\end{array}$ & $\begin{array}{c}0.67 \\
(0.20 \\
2.24)\end{array}$ & $\begin{array}{c}0.83 \\
(0.46 \\
1.53)\end{array}$ & $\begin{array}{c}0.43 \\
(0.08 \\
2.30)\end{array}$ & $\begin{array}{c}0.74 \\
(0.24 \\
2.30)\end{array}$ & $\begin{array}{r}0.75 \\
(0.45 \\
1.48)\end{array}$ & $\begin{array}{c}1.23(0.40 \\
3.75)\end{array}$ & $\begin{array}{c}1.22 \\
(0.38 \\
4.11)\end{array}$ & $\begin{array}{c}0.81 \\
(0.27 \\
2.40)\end{array}$ & $\begin{array}{c}0.28 \\
(0.08, \\
0.91)\end{array}$ & $\begin{array}{c}0.61 \\
(0.27 \\
1.32)\end{array}$ & $\begin{array}{c}0.62 \\
(0.23 \\
1.58)\end{array}$ & $\begin{array}{c}0.81 \\
(0.25 \\
3.17)\end{array}$ & Placebo \\
\hline
\end{tabular}

drugs affecting the structure and function of DNA, including cisplatin, ifosfamide, bleomycin, and mitomycin $\mathrm{C}$, were the most common medication used in cervix cancer treatment, but the specific mechanism of agents differed from each other, such as cisplatin can form a cross link between the guanines in the DNA after dissociation with chlorine $[54,55]$ while ifosfamide lead DNA during $\mathrm{S}$ phase to shape into cross link, which inhibits the growth and reproduction of tumor cells [56]. Antimetabolites, fluorouracil and hydroxyurea involved, are referred to as the drugs influencing biosynthesis of nucleic acid as well as being vital to the cell growth and proliferation $[57,58]$. By distinct mechanisms, the ultimate purpose of both agents is impeding the synthesis of DNA [59-61]. With respect to the drugs acting on the necessary proteins as tubulin and ribosome, paclitaxel and docetaxel upset 
Table 8: SUCRA value for each treatment under the outcomes of recurrence and distant metastasis

\begin{tabular}{lcc}
\hline & \multicolumn{2}{c}{ Outcome } \\
\cline { 2 - 3 } & Recurrence & Distant metastasis \\
\hline Ble + Cis + Ifo & 0.43 & $\mathbf{0 . 8 3}$ \\
Ble + Cis + MitoC + Vbl & 0.24 & 0.20 \\
Ble + Cis + Vbl & 0.21 & 0.56 \\
Ble + Cis + Ver & 0.18 & 0.54 \\
Cis & 0.52 & 0.41 \\
Cis + Doc & $\mathbf{0 . 7 4}$ \\
Cis + Epi & 0.20 & $\mathbf{0 . 7 2}$ \\
Cis + Flu & 0.68 & 0.50 \\
Cis + Flu + Hyd & 0.67 & 0.48 \\
Cis + Ifo & $\mathbf{0 . 9 3}$ & - \\
Cis + Ifo + Pac & $\mathbf{0 . 9 1}$ & - \\
Cis + Methotrexate & 0.38 & - \\
Cis + Mitolactol & 0.47 & 0.23 \\
Cis + Pac & - & - \\
Cis + Topotecan & 0.66 & 0.23 \\
Cis + Ver & 0.26 & - \\
Epi & 0.56 & 0.45 \\
Flu & 0.60 & $\mathbf{0 . 8 8}$ \\
Flu + MitoC & 0.71 & 0.61 \\
Hyd & 0.42 & 0.61 \\
\hline
\end{tabular}

A

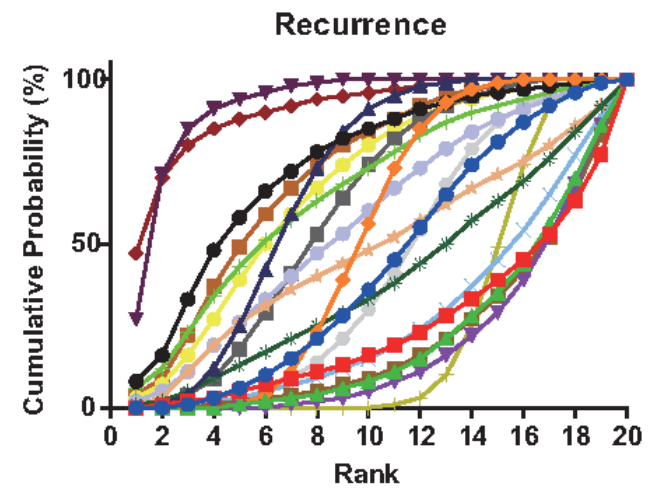

B

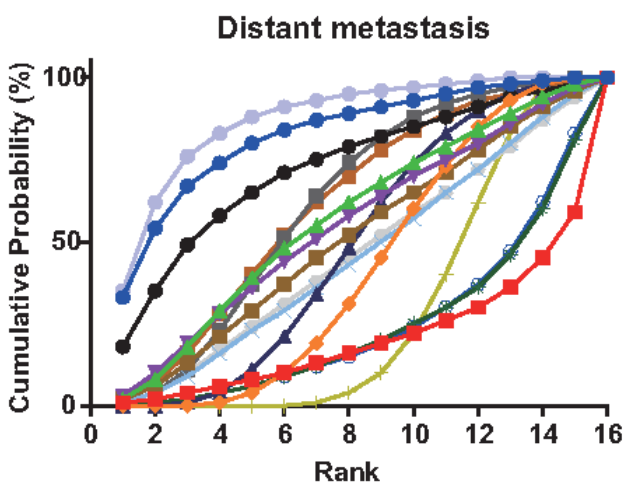

$$
\begin{aligned}
& \rightarrow \text { Ble }+ \text { Cis + Ifo } \rightarrow \text { Cis } \rightarrow \text { Cis + Ifo Cis + Pac } \rightarrow \text { Flu }+\mathrm{MitoC} \\
& \rightarrow \mathrm{Ble}+\mathrm{Cis}+\mathrm{MitoC}+\mathrm{Vbl} \rightarrow \mathrm{Cis}+\mathrm{Doc} \rightarrow \mathrm{Cis}+\mathrm{Ifo}+\mathrm{Pac}+\mathrm{Cis}+\text { Topotecan }-\mathrm{Hyd} \\
& \therefore \mathrm{Ble}+\mathrm{Cis}+\mathrm{Vbl} \quad \rightarrow \mathrm{Cis}+\mathrm{Epi} \# \mathrm{Cis}+\text { Methotrexate } \approx \mathrm{Cis}+\mathrm{Vcr} \rightarrow \mathrm{Flu} \\
& \rightarrow \text { Ble }+ \text { Cis }+ \text { V cr } \quad \rightarrow \text { Cis + Flu } \rightarrow \text { Cis + Mitolactol } \quad-\text { Cis + Flu + Hyd }- \text { Epi }+ \text { Placebo }
\end{aligned}
$$

Figure 2: Cumulative ranking probability curves for recurrence and distant metastasis. (A) recurrence; (B) distant metastasis. 

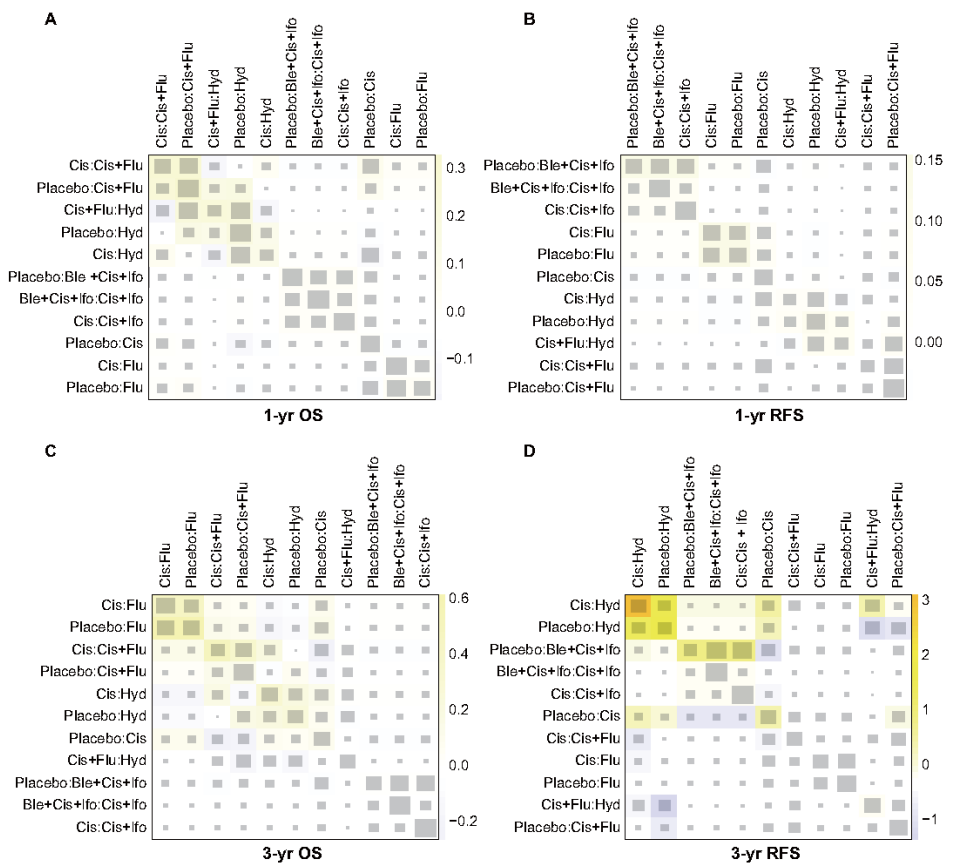

D
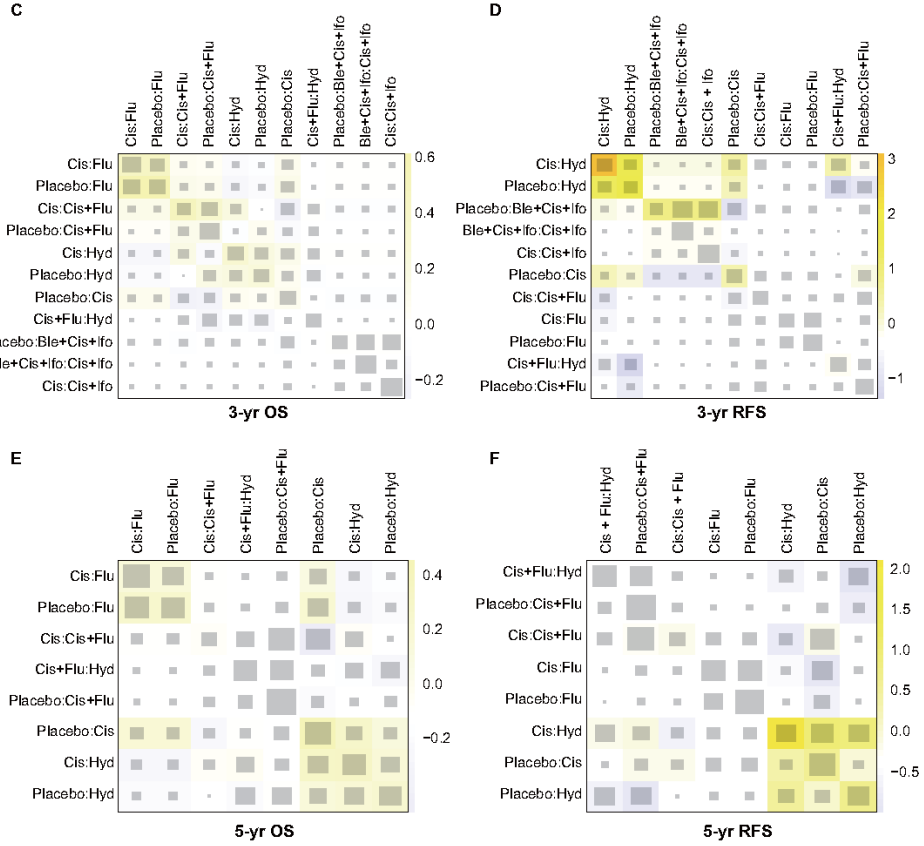

Figure 3: Netheat plots of inconsistency test for survival outcomes. (A) 1-year overall survival (1-year OS); (B) 1-year recurrence-free survival (1year RFS); (C) 3-year OS; (D) 3-year RFS; (E) 5-year OS; (F) 5-year RFS.

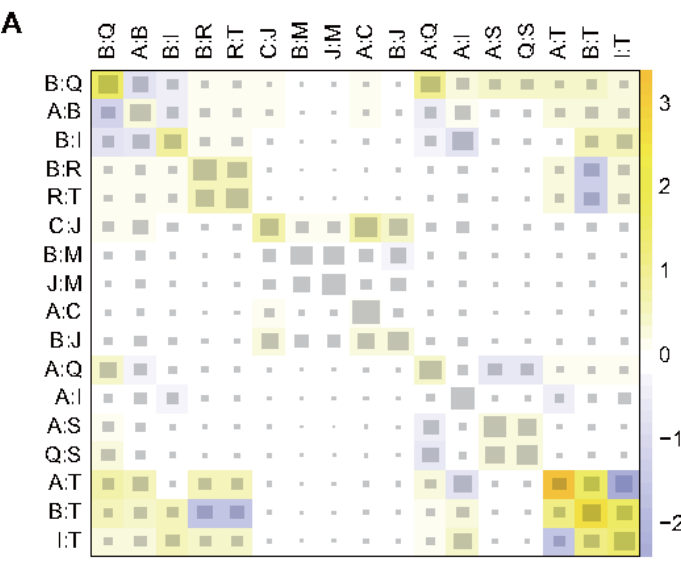

Recurrence

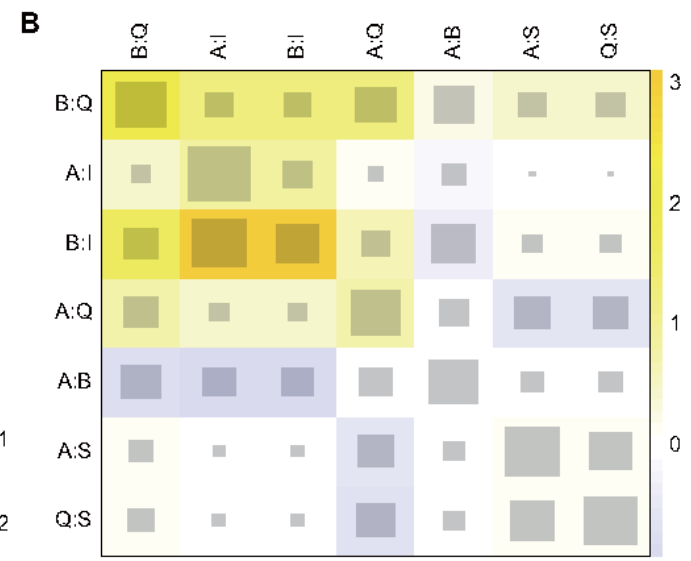

Distant metastas is

A: Placebo B: Cisplatin $\quad$ C: Bleomycin + Cisplatin + lfosfamide $\quad$ D: Bleomycin + Cisplatin + Mitomycin C + Vinblastine $\mathrm{E}$ : Bleomycin + Cisplatin + Vinblastine $\quad \mathrm{F}$ : Bleomycin + Cisplatin + Vincristine $\quad$ G: Cisplatin + Docetaxel $\mathrm{H}$ : Cisplatin + Epirubicin $\quad$ I: Cisplatin + Flurouracil J: Cisplatin + Ifosfamide $\quad \mathrm{K}:$ Cisplatin + Ifosfamide + Paclitaxel L: Cisplatin + Methotrexate $\mathrm{M}$ : Cisplatin + Mitolactol $\mathrm{N}$ : Cisplatin + Topotecan $\mathrm{O}$ : Cisplatin + Vincristine

P: Epirubicin Q: Fluorouracil R: Cisplatin + Fluorouracil + Hydroxyurea S: Fluorouracil + Mitomycin C T: Hydroxyurea

Figure 4: Netheat plots of inconsistency test for recurrence and distant metastasis. (A) recurrence; (B) distant metastasis. 
the dynamic equilibrium between tubulin and its dimer, accelerate the assembling of tubulin and interfere with its disassembly, therefore stop the cell cycle at $\mathrm{G}_{2} / \mathrm{M}$ phase [62-64]. Epirubicin can insert the base pairs of DNA, limiting the activity of transcriptase to influence the synthesis of mRNA, especially in the $\mathrm{S}$ and $\mathrm{M}$ phase of cell cycle [65]. Thus, the combination of multiple drugs with distinctive anti-cancer mechanisms would tend to exhibit superior efficacy especially to reduce the drug resistance during the long term administration.

This NMA assessed the efficacy of 22 chemotherapeutic treatments, containing nearly all clinical regular prescription in the aspects of 1-year, 3-year, and 5-year OS and RFS, as well as the incidence of recurrence and distant metastasis. However, there is still insufficiency that cannot be denied. First, the inclusion trials were limited, and 13 strategies were emerged only once, the inherent error of which were brought in this NMA. Next, the dose and delivery method of each agents were not considered to be an impact factor, but these factors did have influence on their efficacy. The discrepancy on 5-year RFS of the trials between Pearcey and Wong might be caused by the dosage of cisplatin [39]. Moreover, though the FIGO stages of subjects were listed, there was no discrimination in the course of data analysis. Nevertheless, in the clinical practice, the conditions of patients had an important impact on treatment results, including periaortic and pelvic lymph node status, patient age, performance status, bilateral disease and clinical stage [66]. For instance it is evident that the tumor size had the direct relation to the occurrence of metastasis and recurrence [67, 68]. Therefore, more authentic trials are needed and on the basis of colossal data, the particular subgroup analysis can be performed.

Overall, in consideration of the results of this NMA, cisplatin+fluorouracil+hydroxyurea, cisplatin + docetaxel

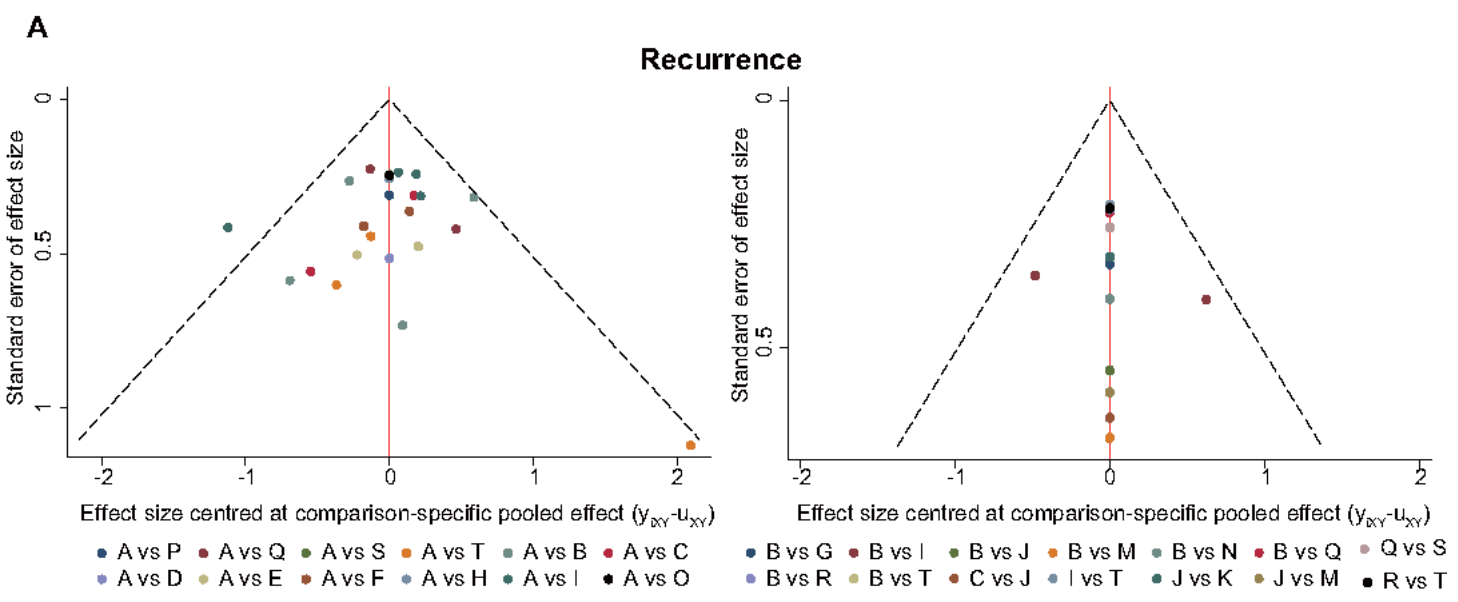

B Distant metastas is
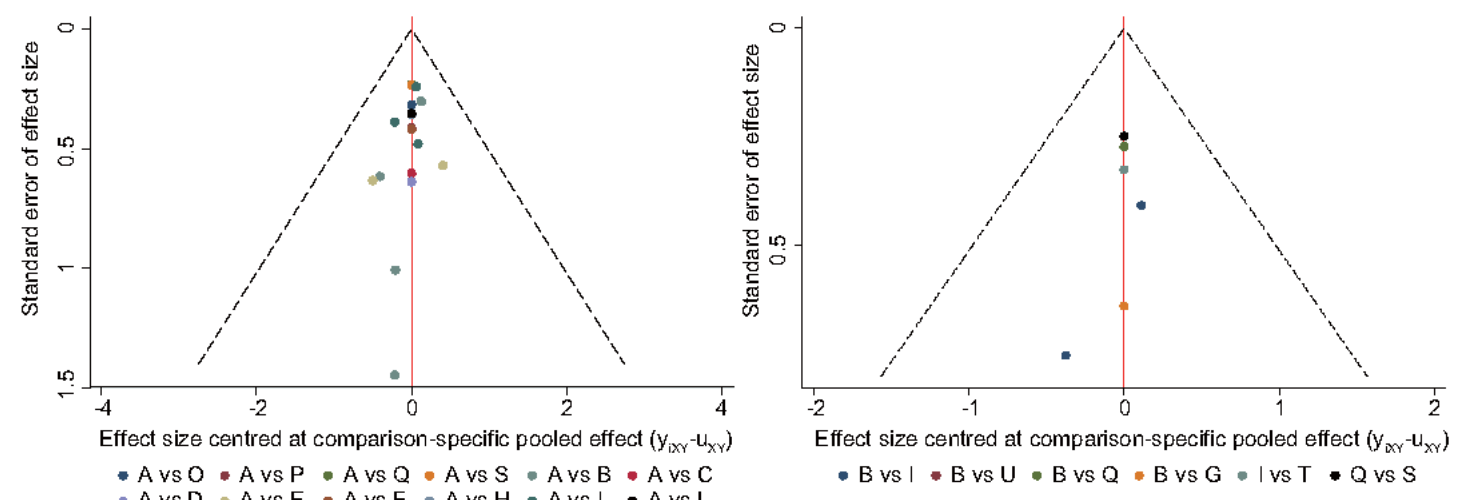

A: Placebo B: Cisplatin C: Bleomycin + Cisplatin + Ifosfamide $\quad$ D: Bleomycin + Cisplatin + Mitomycin C + Vinblastine

$\mathrm{E}$ : Bleomycin + Cisplatin + Vinblastine $\quad \mathrm{F}$ : Bleomycin + Cisplatin + Vincristine $\quad$ G: Cisplatin + Docetaxel

$\mathrm{H}$ : Cisplatin + Epirubicin $\quad$ I: Cisplatin + Flurouracil J: Cisplatin + Ifosfamide $\mathrm{K}$ : Cisplatin + Ifosfamide + Paclitaxel

$\mathrm{L}$ : Cisplatin + Methotrexate M: Cisplatin + Mitolactol $\mathrm{N}$ : Cisplatin + Topotecan $\mathrm{O}$ : Cisplatin + Vincristine

P: Epirubicin Q: Fluorouracil R: Cisplatin + Fluorouracil + Hydroxyurea S: Fluorouracil + Mitomycin C T: Hydroxyurea

Figure 5: Comparison adjusted funnel plots of publication bias test for recurrence and distant metastasis. (A) recurrence; (B) distant metastasis. 
and fluorouracil+mitomycin C, were outstanding in prolonging the length of OS, whilemitomycin $\mathrm{C}$, cispla tin+fluorouracil+hydroxyurea and cisplatin+docetaxel had good performance in RFS. Integrating both OS and RFS data, cisplatin+fluorouracil+hydroxyurea and cisplatin+docetaxel were highly recommended as first tier chemotherapies based on their equally preferable performance in long term. The first three with excellent performance in reducing the recurrence were cisplatin+ifosfamide, cisplatin+ifosfamide+paclitaxel, and cisplatin+docetaxel, in contrast to epirubicin which were beneficial to the significant decrease of distant metastasis. However, it should be noted that the individual conditions of patient should be taken into account thoroughly in clinical application.

\section{MATERIALS AND METHODS}

\section{Search strategy}

To obtain the relevant trial data, we searched electronic database PubMed, Embase and Cochrane Library for RCTs and clinical trials, regardless of the diversity of language, with the following key terms and their synonyms combined, "cervix cancer", "radiotherapy", "surgery", and among "chemotherapy", "concurrent chemotherapy", "neoadjuvant chemotherapy", "adjuvant chemotherapy", and specific drugs, such as "cisplatin", "fluorouracil", "hydroxyurea" were included. Meanwhile, we also examined the reference lists of all the existed meta-analyses and systemic reviews, to guarantee the sample size of tested interventions. And all these work mentioned above were done by two reviewers individually.

\section{Inclusion and exclusion criteria}

All the included trials must meet the listed criteria: (i) at least one of the involved interventions should be used to treat the cervix cancer of patients; (ii) the interventions could include concurrent chemotherapy, neoadjuvant chemotherapy, and adjuvant chemotherapy, but should be combined with radiotherapy and after surgery, while the strategy of radiotherapy and surgery were no limitations; (iii) the overall survival (OS) or the recurrence-free survival (RFS) should be compared between two interventions or interventions and placebo. Besides, although some trials satisfied the inclusion conditions, since they carried out between different methods of administration of the identical drug or the intervention they investigated which cannot form a loop, they were still excluded eventually. The trials included should also meet PRISMA guidelines. According to these criteria, two reviewers screened titles and abstracts of all retrieved articles, and the full texts would be examined respectively when necessary. And any arguments would be solved under discussion by the panel.

\section{Outcome measurements and data extraction}

The basic features of this study, including author, year of publication, country, and the efficacy outcomes, were extracted from each eligible trial [69]. To assess the prognosis of chemotherapy aiming to cervix cancer, OS and RFS are the common outcomes. And quoting the interpretation from the NCI, OS indicates the length of time from the start of the chemotherapy for the cervix cancer, that patients diagnosed with the disease are still alive; and RFS means the length of time during and after the chemotherapy of the cervix cancer, that a patient lives without the diagnosed cancer. Except for the data given directly, the outcomes can also be extracted from the OS and RFS curves, the cumulative percentage versus time after chemotherapy administration. The incidence of recurrence and distant metastases were also evaluated as efficacy predictors.

\section{Statistical analysis}

Based on the connection among treatments, four network plots on recurrence, distant metastasis, OS and RFS were drawn. The heterogeneity of fixed-effects model among each study effect was calculated through Cochran's Q and I squared statistic, which were presented in the net heat plots $[70,71]$. Generally, if $P_{h}<0.05$ or $I^{2}>50 \%$, it implied that a significant heterogeneity was existed, and then the fixed-effects model would be replaced by the random-effects model.

STATA 12.0 software and WinBUGS software were used for, which showed us the combination of direct and indirect evidence. In this NMA, we synthesized the direct and indirect evidence on 1-year, 3-year, 5-year OS and RFS, recurrence and distant metastasis. HR or OR with their $95 \%$ credible intervals (CrIs) were applied to evaluate the relative efficacy for specific comparison. To sort the chemotherapies according to their efficacies on recurrence and distant metastasis, the surface under the cumulative ranking curve (SUCRA) was applied, cumulating the percentages of each intervention with assuming the best, the second best and so on. And then, the optimal treatment would have the highest cumulative probability [72]. Furthermore, the heat plots for each outcome were performed to reflect the contribution of direct evidence to the network comparison and their inconsistency. Moreover, the publication bias on recurrence and distant metastasis were considered through the funnel plots, standard error of effect size versus the effect size centered at comparison-specific pooled effect.

\section{Abbreviations}

HPV (human papillomavirus); RCTs (Randomized controlled trials); OS (overall survival); RFS (recurrencefree survival); HR (hazard ratio); OR (odds ratio); CI 
(confidence interval); SUCRA (surface under cumulative ranking curve); HPV (human papillomavirus); FIGO (Federation of Gynecology and Obstetrics); RCTs (randomized control trials); MA (meta-analyses); NMA (network meta-analysis); dUMP(deoxyuridine monophosphate); CrIs (credible intervals); Ble (Bleomycin); Cis (Cisplatin); Doc (Docetaxel); Epi (Epirubicin); Flu (Fluorouracil); Hyd (Hydroxyurea); Ifo (Ifosfamide); MitoC (Mitomycin C); Pac (Paclitaxel); Vbl (Vinblastine); Vcr (Vincristine).

\section{CONFLICTS OF INTEREST}

The authors declare no financial or other conflicts of interest.

\section{REFERENCES}

1. Torre LA, Bray F, Siegel RL, Ferlay J, Lortet-Tieulent J, Jemal A. Global cancer statistics, 2012. CA Cancer J Clin. 2015; 65:87-108.

2. Miller KD, Siegel RL, Lin CC, Mariotto AB, Kramer JL, Rowland JH, Stein KD, Alteri R, Jemal A. Cancer treatment and survivorship statistics, 2016. CA Cancer J Clin. 2016; 66:271-89.

3. Walboomers JM, Jacobs MV, Manos MM, Bosch FX, Kummer JA, Shah KV, Snijders PJ, Peto J, Meijer CJ, Muñoz N. Human papillomavirus is a necessary cause of invasive cervical cancer worldwide. J Pathol. 1999; 189:12-19.

4. Ferlay J, Soerjomataram I, Dikshit R, Eser S, Mathers C, Rebelo M, Parkin DM, Forman D, Bray F. Cancer incidence and mortality worldwide: sources, methods and major patterns in GLOBOCAN 2012. Int J Cancer. 2015; 136:E359-86.

5. Pisani P, Parkin DM, Bray F, Ferlay J. Estimates of the worldwide mortality from 25 cancers in 1990. Int J Cancer. 1999; 83:18-29.

6. Franco EL, Schlecht NF, Saslow D. The epidemiology of cervical cancer. Cancer J. 2003; 9:348-59.

7. Bosch FX, Manos MM, Muñoz N, Sherman M, Jansen AM, Peto J, Schiffman MH, Moreno V, Kurman R, Shah KV. Prevalence of human papillomavirus in cervical cancer: a worldwide perspective. International biological study on cervical cancer (IBSCC) Study Group. J Natl Cancer Inst. $1995 ; 87: 796-802$.

8. Plummer M, Herrero R, Franceschi S, Meijer CJ, Snijders P, Bosch FX, de Sanjose S, Munoz N and Group IM-cCCS. Smoking and cervical cancer: pooled analysis of the IARC multi-centric case - control study. Cancer Causes Control. $2003 ; 14: 805-814$.

9. International Collaboration of Epidemiological Studies of Cervical Cancer. Cervical carcinoma and reproductive factors: collaborative reanalysis of individual data on
16,563 women with cervical carcinoma and 33,542 women without cervical carcinoma from 25 epidemiological studies. Int J Cancer. 2006; 119:1108-24.

10. Lanciano RM, Won M, Hanks GE. A reappraisal of the International Federation of Gynecology and Obstetrics staging system for cervical cancer. A study of patterns of care. Cancer. 1992; 69:482-87.

11. Beiner ME, Covens A. Surgery insight: radical vaginal trachelectomy as a method of fertility preservation for cervical cancer. Nat Clin Pract Oncol. 2007; 4:353-61.

12. Dische S. Radiotherapy of cervical cancer. Clin Obstet Gynaecol. 1985; 12:203-27.

13. Eifel PJ, Winter K, Morris M, Levenback C, Grigsby PW, Cooper J, Rotman M, Gershenson D, Mutch DG. Pelvic irradiation with concurrent chemotherapy versus pelvic and para-aortic irradiation for high-risk cervical cancer: an update of radiation therapy oncology group trial (RTOG) 90-01. J Clin Oncol. 2004; 22:872-80.

14. Whitney CW, Sause W, Bundy BN, Malfetano JH, Hannigan EV, Fowler WC Jr, Clarke-Pearson DL, Liao SY. Randomized comparison of fluorouracil plus cisplatin versus hydroxyurea as an adjunct to radiation therapy in stage IIB-IVA carcinoma of the cervix with negative paraaortic lymph nodes: a Gynecologic Oncology Group and Southwest Oncology Group study. J Clin Oncol. 1999; 17:1339-48.

15. Kim HS, Sardi JE, Katsumata N, Ryu HS, Nam JH, Chung HH, Park NH, Song YS, Behtash N, Kamura T, Cai HB, Kim JW. Efficacy of neoadjuvant chemotherapy in patients with FIGO stage IB1 to IIA cervical cancer: an international collaborative meta-analysis. Eur J Surg Oncol. 2013; 39:115-124.

16. Rydzewska L, Tierney J, Vale CL, Symonds PR. Neoadjuvant chemotherapy plus surgery versus surgery for cervical cancer. Cochrane Database Syst Rev. 2012; 12:CD007406.

17. Eddy GL, Bundy BN, Creasman WT, Spirtos NM, Mannel RS, Hannigan E, O'Connor D. Treatment of ("bulky") stage IB cervical cancer with or without neoadjuvant vincristine and cisplatin prior to radical hysterectomy and pelvic/paraaortic lymphadenectomy: a phase III trial of the gynecologic oncology group. Gynecol Oncol. 2007; 106:362-69.

18. Yin M, Zhang H, Li H, Li X, Liu Y, Chen X, Lou G, Li $\mathrm{K}$. The toxicity and long-term efficacy of nedaplatin and paclitaxel treatment as neoadjuvant chemotherapy for locally advanced cervical cancer. J Surg Oncol. 2012; 105:206-11.

19. Symonds RP, Habeshaw T, Reed NS, Paul J, Pyper E, Yosef H, Davis J, Hunter R, Davidson SE, Stewart A, Cowie V, Sarkar T. The Scottish and Manchester randomised trial of neo-adjuvant chemotherapy for advanced cervical cancer. Eur J Cancer. 2000; 36:994-1001.

20. Thomas G, Dembo A, Ackerman I, Franssen E, Balogh J, Fyles A, Levin W. A randomized trial of standard versus 
partially hyperfractionated radiation with or without concurrent 5-fluorouracil in locally advanced cervical cancer. Gynecol Oncol. 1998; 69:137-45.

21. Buda A, Fossati R, Colombo N, Fei F, Floriani I, Gueli Alletti D, Katsaros D, Landoni F, Lissoni A, Malzoni C, Sartori E, Scollo P, Torri V, et al. Randomized trial of neoadjuvant chemotherapy comparing paclitaxel, ifosfamide, and cisplatin with ifosfamide and cisplatin followed by radical surgery in patients with locally advanced squamous cell cervical carcinoma: the SNAP01 (Studio Neo-Adjuvante Portio) Italian Collaborative Study. J Clin Oncol. 2005; 23:4137-45.

22. Tattersall MH, Lorvidhaya V, Vootiprux V, Cheirsilpa A, Wong F, Azhar T, Lee HP, Kang SB, Manalo A, Yen MS, Kampono N, Aziz F, and Cervical Cancer Study Group of the Asian Oceanian Clinical Oncology Association. Randomized trial of epirubicin and cisplatin chemotherapy followed by pelvic radiation in locally advanced cervical cancer. J Clin Oncol. 1995; 13:444-51.

23. Tseng CJ, Chang CT, Lai CH, Soong YK, Hong JH, Tang SG, Hsueh S. A randomized trial of concurrent chemoradiotherapy versus radiotherapy in advanced carcinoma of the uterine cervix. Gynecol Oncol. 1997; 66:52-58.

24. Omura GA, Blessing JA, Vaccarello L, Berman ML, Clarke-Pearson DL, Mutch DG, Anderson B. Randomized trial of cisplatin versus cisplatin plus mitolactol versus cisplatin plus ifosfamide in advanced squamous carcinoma of the cervix: a Gynecologic Oncology Group study. J Clin Oncol. 1997; 15:165-71.

25. Bloss JD, Blessing JA, Behrens BC, Mannel RS, Rader JS, Sood AK, Markman M, Benda J. Randomized trial of cisplatin and ifosfamide with or without bleomycin in squamous carcinoma of the cervix: a gynecologic oncology group study. J Clin Oncol. 2002; 20:1832-37.

26. Tattersall MH, Ramirez C, Coppleson M. A randomized trial of adjuvant chemotherapy after radical hysterectomy in stage Ib-IIa cervical cancer patients with pelvic lymph node metastases. Gynecol Oncol. 1992; 46:176-81.

27. Tattersall MH, Ramirez C and Coppleson M. A randomized trial comparing platinum-based chemotherapy followed by radiotherapy vs. radiotherapy alone in patients with locally advanced cervical cancer. International Journal of Gynecological Cancer. 1992; 2:244-251.

28. Tabata T, Takeshima N, Nishida H, Hirai Y, Hasumi K. A randomized study of primary bleomycin, vincristine, mitomycin and cisplatin (BOMP) chemotherapy followed by radiotherapy versus radiotherapy alone in stage IIIB and IVA squamous cell carcinoma of the cervix. Anticancer Research. 2003; 23:2885-2890.

29. Long HJ 3rd, Bundy BN, Grendys EC Jr, Benda JA, McMeekin DS, Sorosky J, Miller DS, Eaton LA, Fiorica JV, and Gynecologic Oncology Group Study. Randomized phase III trial of cisplatin with or without topotecan in carcinoma of the uterine cervix: a Gynecologic Oncology Group Study. J Clin Oncol. 2005; 23:4626-33.

30. Pu J, Qin SS, Ding JX, Zhang Y, Zhu WG, Yu CH, Li T, Tao GZ, Ji FZ, Zhou XL, Han JH, Ji YL, Sun JX. A randomized controlled study of single-agent cisplatin and radiotherapy versus docetaxel/cisplatin and radiotherapy in high-risk early-stage cervical cancer after radical surgery. J Cancer Res Clin Oncol. 2013; 139:703-08.

31. Lanciano R, Calkins A, Bundy BN, Parham G, Lucci JA 3rd, Moore DH, Monk BJ, O'Connor DM. Randomized comparison of weekly cisplatin or protracted venous infusion of fluorouracil in combination with pelvic radiation in advanced cervix cancer: a gynecologic oncology group study. J Clin Oncol. 2005; 23:8289-95.

32. Herod J, Burton A, Buxton J, Tobias J, Luesley D, Jordan S, Dunn J, Poole CJ. A randomised, prospective, phase III clinical trial of primary bleomycin, ifosfamide and cisplatin (BIP) chemotherapy followed by radiotherapy versus radiotherapy alone in inoperable cancer of the cervix. Ann Oncol. 2000; 11:1175-81.

33. Nagai N, Oshita T, Murakami J, Shigemasa K, Hirokawa Y, Ohama K. Radiotherapy combined with transcatheter arterial infusion of cisplatin versus oral fluoropyrimidine anticancer agent for locally advanced carcinoma of the uterine cervix: a prospective follow-up study. Oncol Rep. 2001; 8:119-25.

34. Sundfør K, Tropé CG, Högberg T, Onsrud M, Koern J, Simonsen E, Bertelsen K, Westberg R. Radiotherapy and neoadjuvant chemotherapy for cervical carcinoma. A randomized multicenter study of sequential cisplatin and 5-fluorouracil and radiotherapy in advanced cervical carcinoma stage 3B and 4A. Cancer. 1996; 77:2371-78.

35. Kim YS, Shin SS, Nam JH, Kim YT, Kim YM, Kim JH, Choi EK. Prospective randomized comparison of monthly fluorouracil and cisplatin versus weekly cisplatin concurrent with pelvic radiotherapy and high-dose rate brachytherapy for locally advanced cervical cancer. Gynecol Oncol. 2008; 108:195-200.

36. Pearcey R, Brundage M, Drouin P, Jeffrey J, Johnston D, Lukka H, MacLean G, Souhami L, Stuart G, Tu D. Phase III trial comparing radical radiotherapy with and without cisplatin chemotherapy in patients with advanced squamous cell cancer of the cervix. J Clin Oncol. 2002; 20:966-72.

37. Moore DH, Blessing JA, McQuellon RP, Thaler HT, Cella D, Benda J, Miller DS, Olt G, King S, Boggess JF, Rocereto TF. Phase III study of cisplatin with or without paclitaxel in stage IVB, recurrent, or persistent squamous cell carcinoma of the cervix: a gynecologic oncology group study. J Clin Oncol. 2004; 22:3113-19.

38. Morris M, Eifel PJ, Lu J, Grigsby PW, Levenback C, Stevens RE, Rotman M, Gershenson DM, Mutch DG. Pelvic radiation with concurrent chemotherapy compared with pelvic and para-aortic radiation for high-risk cervical cancer. N Engl J Med. 1999; 340:1137-43. 
39. Wong LC, Choo YC, Choy D, Sham JS, Ma HK. Long-term follow-up of potentiation of radiotherapy by cis-platinum in advanced cervical cancer. Gynecol Oncol. 1989; 35:159-63.

40. Roberts KB, Urdaneta N, Vera R, Vera A, Gutierrez E, Aguilar Y, Ott S, Medina I, Sempere P, Rockwell S, Sartorelli AC, Fischer DB, Fischer JJ. Interim results of a randomized trial of mitomycin $\mathrm{C}$ as an adjunct to radical radiotherapy in the treatment of locally advanced squamous-cell carcinoma of the cervix. Int J Cancer. 2000; 90:206-23.

41. Leborgne F, Leborgne JH, Doldán R, Zubizarreta E, Ortega B, Maisonneuve J, Musetti E, Hekimian L, Mezzera J. Induction chemotherapy and radiotherapy of advanced cancer of the cervix: a pilot study and phase III randomized trial. Int J Radiat Oncol Biol Phys. 1997; 37:343-50.

42. Piver MS, Barlow JJ, Vongtama V, Blumenson L. Hydroxyurea: a radiation potentiator in carcinoma of the uterine cervix. A randomized double-blind study. Am J Obstet Gynecol. 1983; 147:803-08.

43. Piver MS, Vongtama V, Emrich LJ. Hydroxyurea plus pelvic radiation versus placebo plus pelvic radiation in surgically staged stage IIIB cervical cancer. J Surg Oncol. 1987; 35:129-34.

44. Hreshchyshyn MM, Aron BS, Boronow RC, Franklin EW 3rd, Shingleton HM, Blessing JA. Hydroxyurea or placebo combined with radiation to treat stages IIIB and IV cervical cancer confined to the pelvis. Int J Radiat Oncol Biol Phys. 1979; 5:317-22.

45. Donnelly ED, Refaat T, Gentile M, Herskovic A, Boyle J, Helenowski I, Rademaker A, Lurain J, Schink J, Singh D, Strauss JB, Small W Jr. Evaluation of Outcomes in Patients With Carcinoma of the Cervix Treated With Concurrent Radiation and Cisplatin Versus Cisplatin/5-FU Compared With Radiation Alone. Am J Clin Oncol. 2015; $38: 437-41$.

46. Lorvidhaya V, Chitapanarux I, Sangruchi S, Lertsanguansinchai P, Kongthanarat Y, Tangkaratt S, Visetsiri E. Concurrent mitomycin C, 5-fluorouracil, and radiotherapy in the treatment of locally advanced carcinoma of the cervix: a randomized trial. Int J Radiat Oncol Biol Phys. 2003; 55:1226-32.

47. Rose PG, Bundy BN, Watkins EB, Thigpen JT, Deppe G, Maiman MA, Clarke-Pearson DL, Insalaco S, Mackey D. Concurrent cisplatin-based radiotherapy and chemotherapy for locally advanced cervical cancer. N Engl J Med. 1999; 340:1144-53.

48. Peters WA 3rd, Liu PY, Barrett RJ 2nd, Stock RJ, Monk BJ, Berek JS, Souhami L, Grigsby P, Gordon W Jr, Alberts DS. Concurrent chemotherapy and pelvic radiation therapy compared with pelvic radiation therapy alone as adjuvant therapy after radical surgery in high-risk early-stage cancer of the cervix. J Clin Oncol. 2000; 18:1606-13.

49. Keys HM, Bundy BN, Stehman FB, Muderspach LI, Chafe WE, Suggs CL 3rd, Walker JL, Gersell D. Cisplatin, radiation, and adjuvant hysterectomy compared with radiation and adjuvant hysterectomy for bulky stage IB cervical carcinoma. N Engl J Med. 1999; 340:1154-61.

50. Nedovic J, Protrka Z, Ninkovic S, Mitrovic S, Vojinovic R, Glisic J, Markovic-Filipovic B, Milosevic B, Peulic M, Cvetkovic A. Cisplatin monotherapy with concurrent radiotherapy versus combination of cisplatin and 5-fluorouracil chemotherapy with concurrent radiotherapy in patients with locoregionally advanced cervical carcinoma. Journal of BUON. 2012; 17:740-745.

51. Kumar L, Kaushal R, Nandy M, Biswal BM, Kumar S, Kriplani A, Singh R, Rath GK, Kochupillai V. Chemotherapy followed by radiotherapy versus radiotherapy alone in locally advanced cervical cancer: a randomized study. Gynecol Oncol. 1994; 54:307-15.

52. Wong LC, Ngan HY, Cheung AN, Cheng DK, Ng TY, Choy DT. Chemoradiation and adjuvant chemotherapy in cervical cancer. J Clin Oncol. 1999; 17:2055-60.

53. Garipağaoğlu M, Kayikçioğlu F, Köse MF, Adli M, Gülkesen KH, Koçak Z, Tulunay G. Adding concurrent low dose continuous infusion of cisplatin to radiotherapy in locally advanced cervical carcinoma: a prospective randomized pilot study. Br J Radiol. 2004; 77:581-87.

54. Fuertes MA, Castilla J, Alonso C, Pérez JM. Cisplatin biochemical mechanism of action: from cytotoxicity to induction of cell death through interconnections between apoptotic and necrotic pathways. Curr Med Chem. 2003; 10:257-66.

55. Kikuchi Y. [The mechanism of cisplatin-resistance in ovarian cancer]. Hum Cell. 2001; 14:115-33. The mechanism of cisplatin-resistance in ovarian cancer.

56. Wagner T. Ifosfamide clinical pharmacokinetics. Clin Pharmacokinet. 1994; 26:439-56.

57. Longley DB, Harkin DP, Johnston PG. 5-fluorouracil: mechanisms of action and clinical strategies. Nat Rev Cancer. 2003; 3:330-38.

58. Parker WB, Cheng YC. Metabolism and mechanism of action of 5-fluorouracil. Pharmacol Ther. 1990; 48:381-95.

59. Yarbro JW. Mechanism of action of hydroxyurea. Semin Oncol. 1992 (Suppl 9); 19:1-10.

60. Charache S. Mechanism of action of hydroxyurea in the management of sickle cell anemia in adults. Semin Hematol. 1997 (Suppl 3); 34:15-21.

61. Yarbro JW. Further studies on the mechanism of action of hydroxyurea. Cancer Res. 1968; 28:1082-87.

62. Mozzetti S, Ferlini C, Concolino P, Filippetti F, Raspaglio G, Prislei S, Gallo D, Martinelli E, Ranelletti FO, Ferrandina G, Scambia G. Class III beta-tubulin overexpression is a prominent mechanism of paclitaxel resistance in ovarian cancer patients. Clin Cancer Res. 2005; 11:298-305.

63. Sengupta S, Boge TC, Georg GI, Himes RH. Interaction of a fluorescent paclitaxel analogue with tubulin. Biochemistry. 1995; 34:11889-94.

64. Hasegawa S, Miyoshi Y, Egawa C, Ishitobi M, Taguchi T, Tamaki Y, Monden M, Noguchi S. Prediction of response 
to docetaxel by quantitative analysis of class I and III betatubulin isotype mRNA expression in human breast cancers. Clin Cancer Res. 2003; 9:2992-2997.

65. Tjuljandin SA, Doig RG, Sobol MM, Watson DM, Sheridan WP, Morstyn G, Mihaly G, Green MD. Pharmacokinetics and toxicity of two schedules of high dose epirubicin. Cancer Res. 1990; 50:5095-101.

66. Stehman FB, Bundy BN, DiSaia PJ, Keys HM, Larson JE, Fowler WC. Carcinoma of the cervix treated with radiation therapy. I. A multi-variate analysis of prognostic variables in the Gynecologic Oncology Group. Cancer. 1991; 67:2776-85.

67. Zaino RJ, Ward S, Delgado G, Bundy B, Gore H, Fetter G, Ganjei P, Frauenhoffer E. Histopathologic predictors of the behavior of surgically treated stage IB squamous cell carcinoma of the cervix. A Gynecologic Oncology Group study. Cancer. 1992; 69:1750-58.

68. Delgado G, Bundy B, Zaino R, Sevin BU, Creasman WT, Major F. Prospective surgical-pathological study of disease-free interval in patients with stage IB squamous cell carcinoma of the cervix: a Gynecologic Oncology Group study. Gynecol Oncol. 1990; 38:352-57.

69. Basch E, Reeve BB, Mitchell SA, Clauser SB, Minasian LM, Dueck AC, Mendoza TR, Hay J, Atkinson TM, Abernethy AP, Bruner DW, Cleeland CS, Sloan JA, et al. Development of the National Cancer Institute's patientreported outcomes version of the common terminology criteria for adverse events (PRO-CTCAE). J Natl Cancer Inst. 2014; 106:dju244.

70. Higgins JP, Thompson SG. Quantifying heterogeneity in a meta-analysis. Stat Med. 2002; 21:1539-58.

71. Higgins JP, Thompson SG, Deeks JJ, Altman DG. Measuring inconsistency in meta-analyses. BMJ. 2003; 327:557-60.

72. Rücker G, Schwarzer G. Ranking treatments in frequentist network meta-analysis works without resampling methods. BMC Med Res Methodol. 2015; 15:58. 